\title{
Tissue fluidification promotes a cGAS/STING- mediated cytosolic DNA response in invasive breast cancer
}

\section{Emanuela Frittoli}

ondazione Istituto FIRC di Oncologia Molecolare

\section{Andrea Palamidessi}

Fondazione Istituto FIRC di Oncologia Molecolare https://orcid.org/0000-0002-7134-7023

Fabio lannelli

The FIRC Institute of Molecular Oncology, IFOM, Milano

Federica Zanardi

FIRC Institute of Molecular Oncology

Stefano Villa

University iof Milan

Leonardo Barzaghi

IFOM https://orcid.org/0000-0002-3174-6441

Hind Ando

IFOM

Valeria Cancila

University of Palermo

Galiba Beznuskenko

IFOM

Giulia Della Chiara

IFOM

Massimiliano Pagani

IFOM

Chiara Malinverno

IFOM

Dipanjan Bhattacharya

IFOM

Federica Pisati

IFOM

\section{Weimiao Yu}

Institute of Molecular and Cell Biology https://orcid.org/0000-0001-5092-1009

\section{Viviana Galimberti}


IEO

\title{
Giuseppina Bonizzi
}

IEO

\section{Emanuele Martini}

Italian Foundation for Cancer Research https://orcid.org/0000-0002-3375-7726

\author{
Alexander Mironov \\ FIRC Institute of Molecular Oncology \\ ubaldo gioia \\ IFOM
}

Fabrizio D'adda di fagagna

IFOM

\section{Chiara Rossi}

University of Pavia

Giovanni Bertalot

APPS

\section{Marco Lucioni}

University of Pavia

richard tancredi

University of Pavia

paolo Pedrazzoli

University of Pavia

andrea Vecchione

University of Rome, La Sapienza

Cristiuano Perini

IFOM

FRancesco Ferrari

IFOM

Chiara Lanzuolo

ingm

Guilherme Nader

Curie Ist

Marco Foiani

IFOM

\section{Matthieu Piel}

Institut Curie https://orcid.org/0000-0002-2848-177X

\section{Roberto Cerbino}

University of Milan

\section{Fabio Giavazzi}

University of Milan https://orcid.org/0000-0003-4930-0592 


\section{Claudio Tripodo}

University of Palermo

Giorgio Scita ( $\nabla$ giorgio.scita@ifom.eu )

FIRC Institute of Molecular Oncology https://orcid.org/0000-0001-7984-1889

\section{Article}

Keywords: tissue fluidification, breast cancer, cell density

Posted Date: September 20th, 2021

DOl: https://doi.org/10.21203/rs.3.rs-845173/v1

License: (c) (1) This work is licensed under a Creative Commons Attribution 4.0 International License. Read Full License 


\title{
Tissue fluidification promotes a cGAS/STING-mediated cytosolic DNA response in invasive breast cancer
}

Emanuela Frittoli ${ }^{1, \dagger}$, Andrea Palamidessi ${ }^{1, \dagger}$, Fabio lannelli ${ }^{1}$, Federica Zanardi ${ }^{1}$, Stefano Villa ${ }^{2}$, Leonardo Barzaghi $^{1}$, Hind Abdo ${ }^{1}$, Valeria Cancila ${ }^{3}$, Galina V. Beznuskenko ${ }^{1}$, Giulia della Chiara ${ }^{1}$, Massimiliano Pagani $^{1}{ }^{1}$, Chiara Malinverno ${ }^{1}$, Dipanjan Bhattacharya ${ }^{1}$, Federica Pisati ${ }^{1}$, Weimiao $\mathrm{Yu}^{4}$, Viviana Galimberti ${ }^{5}$, Giuseppina Bonizzi ${ }^{5}$, Emanuele Martini ${ }^{1}$, Alexandre Mironov ${ }^{1}$, Ubaldo Gioia ${ }^{1}$, Fabrizio d'Adda di Fagagna ${ }^{1,6}$, Chiara Rossi ${ }^{7}$, Marco Lucioni ${ }^{7}$, Richard Tancredi ${ }^{8}$, Paolo Pedrazzoli ${ }^{8,9}$, Andrea Vecchione ${ }^{10}$, Cristiano Petrini $^{1}$, Francesco Ferrari ${ }^{1,6}$, Chiara Lanzuolo ${ }^{11}$, Guilherme Nader ${ }^{12}$, Marco Foiani ${ }^{1,13}$, Matthieu Piel ${ }^{12}$, Roberto Cerbino $^{2,}$ ๆ, Fabio Giavazzi ${ }^{* 1,2}$, Claudio Tripodo ${ }^{* 1,3}$ and Giorgio Scita ${ }^{* 1,13}$

${ }^{1}$ IFOM, the FIRC Institute of Molecular Oncology, Via Adamello 16, 20139, Milan, Italy

2 Department of Medical Biotechnology and Translational Medicine, University of Milan, 20090 Segrate, Italy

${ }^{3}$ Department of Health Sciences, Human Pathology Section, University of Palermo School of Medicine, Via del Vespro 129, 90127, Palermo, Italy

${ }^{4}$ Institute of Molecular and Cell Biology, A*STAR, Singapore, \& Bioinformatics Institute, A*STAR, Singapore ${ }^{5}$ European Institute of Oncology (IEO) IRCCS, Via Ripamonti 435, 20141, Milan, Italy

${ }^{6}$ Institute of Molecular Genetics, National Research Council, Pavia, Italy.

7 Unit of Anatomic Pathology, Department of Molecular Medicine, Fondazione IRCCS Policlinico San Matteo, University of Pavia, Pavia, Italy

${ }^{8}$ Medical Oncology Unit, Fondazione IRCCS Policlinico San Matteo, Pavia Italy

${ }^{9}$ Department of Internal Medicine and Medical Therapy, University of Pavia, Pavia, Italy

${ }^{10}$ Department of Clinical and Molecular Medicine, University of Roma, La Sapienza, Romer, Italy.

${ }^{11}$ Institute of Biomedical Technologies, National Research Council, Milan, Italy.

${ }^{12}$ Institut Curie and Institut Pierre Gilles de Gennes, PSL Research University, CNRS, UMR-144, Paris, France

${ }^{13}$ Department of Oncology and Haemato-Oncology, University of Milan, Via Santa Sofia 9/1, 20122, Milan, Italy

Institut Curie and Institut Pierre Gilles de Gennes, PSL Research University, CNRS, UMR-144, Paris, France

П Present address: Faculty of Physics of the University of Vienna, Austria.

${ }^{\dagger}$ These authors contributed equally.

${ }^{*}$ Corresponding authors- these authors contributed equally to the supervision of the work:

Giorgio.scita@ifom.eu, Fabio.giavazzi@unimi.it, claudio.tripodo@unipa.it.

Lead contact: Giorgio Scita, email:giorgio.scita@ifom.eu

\begin{abstract}
The process in which locally confined epithelial malignancies progressively evolve into invasive cancers is often promoted by unjamming, a phase transition from a solid-like to a liquid-like state that occurs in various tissues. Whether this tissue-level mechanical transition impact phenotypes during carcinoma progression remains unclear. We show, here that the large fluctuations in cell density that accompany unjamming result in repeated mechanical deformations of cells and nuclei. Cells react to these protracted mechanical stresses by mounting a mechano-protective response that includes enlarged nuclear size and rigidity, altered heterochromatin distribution, and the remodeling of the perinuclear actin architecture into actin rings. The chronic strains and stresses associated with unjamming together with the reduction of Lamin B1 levels eventually result in DNA damage and nuclear envelope ruptures, with the release of cytosolic DNA that activates a cGAS/STING-dependent cytosolic DNA response gene program. This mechanically-driven transcriptional rewiring ultimately results in a change in cell state, with the emergence of malignant traits, including epithelial-to-mesenchymal plasticity phenotypes and chemo-resistance in invasive breast carcinoma.
\end{abstract}

One-Sentence Summary: A solid-to-fluid phase transition promotes a pro-inflammatory transcriptional response in invasive breast carcinoma 


\section{Introduction and Results}

The mechanical and dynamic properties of cells and tissues are emerging as pivotal regulators of cell behavior and fate in physiology and pathology. During carcinogenesis, cancer cells must constantly deal with and adapt to variable mechanical stress, which, in turn, impacts their phenotypes ${ }^{1}$. Normal epithelial tissues frequently evolve into solid or jammed masses that are densely packed with cancer cells. To become malignant, a certain degree of fluidity is required for a tissue to be able to proliferate, migrate and disseminate. A recently discovered and less understood process by which cells can acquire migratory behavior is cellular unjamming, a phase transition characterized by collective and cooperative cellular motion akin to fluid flow ${ }^{2-9}$. Whether and how unjamming impacts the acquisition of heritable changes that influence tissue state and malignant progression remains unclear.

Ductal adenocarcinoma in situ (DCIS), a precursor of invasive breast cancer, is a remarkable case in point. Firstly, DCISs typically grow at high density within the confinement of the mammary duct lumina, where they may display a highly cohesive growth associated with extreme cell packing and density (e.g., comedonic growth). These conditions expose DCIS to overcrowding and compressive, mechanical stresses that impact their physical state favoring a transition to a solid (jammed) and kinetically-arrested, state. Consistently, nearly $70 \%$ of DCIS will not progress, behaving as indolent lesions, which suggests that packing and extreme confinement do exert tumor-suppressive functions. However, $30 \%$ of these cancers can overcome the caging imposed by the crowded cellular landscape of packed DCIS, by undergoing a solid (jammed)-to-liquid (unjammed) phase transition, which facilitates the acquisition of cell locomotion and progression to invasive ductal carcinoma (IDC).

We hypothesize that this material-like phase transition is a rapid, short-term adaptive response to mechanical challenging conditions that in addition to promoting collective dissemination of early lesions would also coincidentally result in a long-term, transcriptional-dependent phenotype switch in invasive breast carcinoma.

\section{Endocytic-driven tissue fluidification rewires transcription by promoting a cytosolic DNA response gene signature.}

We have recently shown that the expression of the small $G$ protein RAB5A, a pivotal regulator of endosome biogenesis upregulated in human breast cancer and associated with decreased disease-free survival ${ }^{10}$, is sufficient to overcome kinetic and proliferation arrest in both normal and tumorigenic epithelia ${ }^{2,8}$. RAB5A expression does so by triggering a mechanically-driven phase transition from a solid (or jammed) and immobile state to a flocking fluid hyper-locomotory state that is analogous to animal flocking $8,11,12$. This transition is characterized by the coexistence of long-range, coordinated motion and local liquid-like cell rearrangements ${ }^{2}, 8,11-13$. In the context of breast carcinoma, tissue fluidification-via-flocking promotes collective motility and local invasiveness of ductal breast carcinoma ${ }^{2}$. We posit that this mechanically-driven solid-to-fluid transition might also rewire the transcriptional state of early indolent lesion promoting a whole phenotypic switch that impacts tumor progression.

To address this possibility, we began by examining the transcriptional profile of densely packed epithelial monolayers formed by quasi-normal MCF10A cells and the respective oncogenic variant MCF10.DCIS.com cells. Both cell lines were engineered to express RAB5A in a doxycycline-inducible fashion to levels like those found in human invasive breast cancer ${ }^{2}{ }^{10}$. MCF10.DCIS.com cells express oncogenic T24-H-RAS and are used as models for the progression of ductal carcinoma in situ (DCIS) to invasive ductal carcinoma $(\text { IDC) })^{14,15}$.

As expected, MCF10.DCIS.com cells plated as confluent monolayers are jammed and kinetically arrested $^{2,8}$. Induction of RAB5A in both MCF10A and MCF10.DCIS.com jammed monolayers promoted the reawakening of collective motion via flocking ${ }^{2,8}$. This was accompanied by robust and highly reproducible alterations in the transcriptional profile (Fig. $1 \mathrm{~A}$ and Fig. S1A-C). Unexpectedly, gene set enrichment analysis revealed the interferon-stimulated gene signature (ISG) as the most significantly enriched in deregulated genes (Fig. 1C), albeit interferons (alpha, beta, or gamma) and the corresponding interferon receptor genes were marginally affected (Fig. S1D). This suggests that RAB5A-mediated fluidization promotes the rewiring toward an interferon-dependent signature without directly modulating the expression of these cytokines. Noticeably, innate immune responses are also promoted by free endogenous DNA present in the cytosol which is recognized as nonself ${ }^{16}$. We thus tested whether RAB5A expression boosted the cytosolic DNA response (CytoDR) program (Fig. 1C). Determination of the mRNA levels of the selected most upregulated genes by RT-qPCR or immunoblotting confirmed the effect of RAB5A-fluidification. It further highlighted the massive increase in the expression of a number of these genes, which were induced 
by more than 100 to 1000 folds with respect to doxycycline control cells (Fig. 1D-E). The upregulation of ISG was also detected in densely packed fluidized MCF10A monolayers by RNA-seq experiments (Fig. S1A-B) and in MCF10.DCIS.com cells grown as tumoroid in low attachment by quantitative PCR of a set of selected genes (Fig. S2A). In all these conditions, we have previously shown that RAB5A promotes a solid-to-liquid transition via flocking and persistent rotational collective motion ${ }^{2,8}$. RAB5A expression was sufficient to induce a set of CytoDR genes also in SUM-225, an estrogen, progesterone receptor-negative, and HER2-positive breast carcinoma cell line ${ }^{17}$, and in the murine mammary cancer line 4T1 ${ }^{18}$ (Fig. S2A).

Thus, RAB5A induction results in an extended transcriptional rewiring toward a cytosolic gene response state in a variety of fluidized normal and tumorigenic epithelial collectives.

The analysis of CytoDR gene expression in single isolated cells revealed that RAB5A only mildly upregulated CytoDR (Fig. S2B), suggesting that RAB5A-mediated transcriptional rewiring is an emergent property of epithelial cell collectives and associated with tissue fluidification. Consistently, the induction of flocking motion via exposure to a hypotonic solution, which promotes tissues fluidification independently from RAB5A expression ${ }^{2,8}$, was sufficient to increase CytoDR gene expression in a time-dependent fashion (Fig. S2C and Movie S1). Importantly, the concomitant induction of RAB5A-expression and hypotonic treatment synergically activated flocking fluid motility, as revealed by the increase in typical quantities that exemplify collective motility: the average migration speed $v_{m}$, in the velocity correlation length $L_{C}$ and in the root mean square amplitude of the velocity fluctuations $v_{r m s}$ (Fig. S2D and Movie S1). The velocity correlation length $L_{C}$ is extracted from the spatial correlation function $C_{v v}(r)$ of the vectorial velocity, and quantifies the degree of coordination of the collective cellular motion in terms of the size of a group of cells coherently migrating in the same direction. $V_{r m s}$ accounts, instead, for mutual, fluid-like, cell displacements (see Methods for details). The synergic increase in collective motility observed under these conditions also resulted in robust induction of CytoDR gene expression (Fig. S2E). Of note, prolonged hypotonic treatment elicited cellular changes similar to the ones induced upon RAB5A expression, including junctional straightening, increased monolayer rigidity ${ }^{2}, 8$, and elevation of ERK1/2 activity (Fig. S2F). Endocytic dependent tissue fluidification-via-flocking depends on EGFR activation and internalization into the endosomal compartment which acts as a signaling platform for the activation of ERK1/2 signaling axis's, 8 . Treatment with Dynasore to prevent dynamin-dependent internalization or MEK inhibitor to block ERK1/2 activation effectively prevented RAB5A-mediated flocking ${ }^{2,8}$ and impaired CytoDR elevation (Fig. S3A). Finally, neither the induction of RAB5B nor $\mathrm{C}$, which are two highly related members of the RAB5 protein family, promoted unjamming-via-flocking ${ }^{2}$ or elicited CytoDR response (Fig. S3B).

We also studied HaCat keratinocyte cells. These cells undergo flocking after induction of RAB5 $A^{8}$, which is greatly enhanced following the addition of EGF to quiescent, serum-starved cells ${ }^{19}$ (Fig. S3C-E and Movie S2). EGF addition promoted robust flocking, with millimeter-scale correlations in the migration velocities (Fig. S3C-D) but it was insufficient to induce CytoDR genes. CytoDR genes induction required the concomitant expression of RAB5A, which boosted tissue fluidization by greatly enhancing the amplitude of local velocity fluctuations associated with mutual cell displacements (Fig. S3E-F).

All in all, these results indicate that endocytic-mediated tissue fluidization occurring via flocking is capable of transcriptionally rewiring cell collectives toward a cytosolic DNA response in a variety of normal and tumorigenic epithelia.

Tissue fluidification promotes a cytosolic DNA gene response through the cGAS/STING pathway. Cyclic GMP-AMP synthase (cGAS) is an innate immune sensor of DNA that recognizes cytosolic DNA, resulting in the activation of the signaling adaptor stimulator of interferon genes (STING) and its translocation to the Golgi compartment. At this site, STING activates TANK binding kinase 1 (TBK1) to phosphorylate the transcription factor interferon regulatory factor 3 (IRF3), promoting its nuclear translocation and the expression of type I/III interferon and interferon-stimulated genes ${ }^{20}$. cGAS recognizes cytosolic DNA derived from invading microbes ${ }^{21,22} 23$ and also self-DNA from engulfed tumor cells ${ }^{24}$, damaged mitochondria ${ }^{25-27}$, nuclear DNA damage ${ }^{27-31}$.

To determine the involvement of the cGAS/STING axis in the activation of CytoDR due to RAB5Amediated tissue fluidification, we utilized a set of pharmacological and molecular genetic loss-of-function approaches targeting each component of the cGAS/STING/TBK1/IRF3 pathway. Specifically, we silenced cGAS, STING, or IRF3 or treated cells with the cGAS inhibitor, RU.521, the STING antagonist, H-151, or the TBK1/IKK inhibitor, MRT67307, which impairs the phosphorylation of IRF332. All these treatments robustly hampered the upregulation of CytoDR genes induced by tissue fluidification in MCF10.DCIS.com model tissues (Fig. 2A-B and Fig. S4A). We also targeted key transcription factors acting downstream of the cGAS/STING axis, IRF9, STAT1, and STAT2, which robustly reduced CytoDR gene upregulation (Fig. 
2C and Fig. S4A). Immunoblotting of total cellular lysates of densely packed monolayers revealed that IRF3 and both the total and phosphorylated levels of STAT1, but not of CGAS or STING were elevated (Fig. 2D), consistent with this pathway being activated by RAB5A-mediated fluidification of MCF10.DCIS.com cell collectives.

The enzymatic activity of cGAS is activated by double-stranded DNA that leaks or into the cytoplasm from variable sources, including ruptured micronuclei, damaged mitochondria, or fragile and mechanically damaged nuclei. Nuclear damage frequently arises as a consequence of mechanicallyinduced deformation ${ }^{33-37}$. We found no evidence of the altered number of micronuclei (Fig. S4B). We noticed, instead, that fluidification-via-flocking induced by RAB5A is accompanied by large fluctuation in cell density ${ }^{12}$ and cell area (Fig. 2E-G and Movie S3), which we hypothesized to be also associated with nuclear deformations. To quantify these deformations, we developed an automated image analysis pipeline to monitor nuclear shape changes over time. We analyzed control and RAB5-expressing MCF10A and MCF10.DCIS.com monolayers. In these cases, tissue fluidification-via-flocking resulted in significantly larger and faster deformations (Movie S4), which were quantified by measuring the mean squared nuclear strain $\operatorname{MSS}(\tau) \equiv\left\langle\left\langle\Delta a_{n}{ }^{2}(\tau \mid t)\right\rangle_{n}\right\rangle_{t}$ for different delay times $\tau$ and extracting the corresponding strain rate $\dot{\gamma}_{N} \cong$ $M S S(\tau) / \tau$ (Fig. 2H-I). In previous expressions, $\Delta a_{n}(\tau \mid t) \equiv\left[A_{n}(t+\tau)-A_{n}(t)\right] /\left\langle A_{n}(t)\right\rangle_{t}$, where $A_{n}(t)$ is the projected area of the $n$-th nucleus at time $t$ and the symbols $\langle\cdot\rangle_{n}$ and $\langle\cdot\rangle_{t}$ indicate averages performed over all the segmented nuclei and over time, respectively. The increased nuclear strain is the consequence of elevated mechanical stress. Consistently, the mechanosensory and transducer co-transcriptional regulator, YAP1, which is kept off and cytoplasmic-restricted in dense and compact monolayers ${ }^{38}$, accumulated into nuclei to activate a subset of canonical YAP1 target genes in fluidized cell collectives (Fig. S4C-E). We also detected YAP1 reactivation in ex vivo organotypic tumour slices from orthotopically-injected MCF10.DCIS.com cells (Fig. S4F-G). The induction of RAB5A expression in these cells triggers large collective and invasive motility flow ${ }^{2}$. Thus, endocytic fluidification via flocking imposes large strain and mechanical stress that is directly transferred to cell nuclei.

We also noticed that the expression of RAB5A, but not of RAB5B or RAB5C (not shown), in dense monolayers resulted in a significant reduction of the mRNA levels of Lamin B1, but not of Lamin A/C, accompanied by a significant inhibition of the protein levels (Fig. 2J-K). Thus, we reasoned that the increased mechanical stress and reduced Lamin B1 levels might compromise nuclear integrity and result in more frequent ruptures of the nuclear envelope (NE). These events might lead to the exposure of DNA to the cytoplasm that, in turn, can trigger cGAS activation. We verified this possibility in multiple and independent ways.

Firstly, we expressed cGAS-fused to EGFP (EGFP-cGAS) and monitored its localization and distribution. In dense, kinetically- and proliferation-arrested MCF10.DCIS.com monolayers, EGFP-cGAS displayed a primarily cytoplasmic diffuse staining, as expected ${ }^{23}, 39$. Laser-mediated ruptures of the NE caused the rapid and focalized accumulation of cGAS at the site of NE damage (Fig. S5A and Movie S5). Similar focalized perinuclear restricted localization was seen after RAB5A-induction in flocking fluid monolayers (Fig. 3A-B).

Secondly, by measuring the amount of cGAMP, the product of cGAS enzymatic activity ${ }^{23}, 40,41$, we showed that RAB5A-flocking fluid cells exhibited a significant increase in cGAMP levels (Fig. 3C).

Thirdly, we monitored in real-time nuclear envelope ruptures through the analysis of the dynamic distribution of the 3NLS-GFP sensor. As shown in Fig. 3D-E, while control cells display a nuclear restricted expression of 3NLS-GFP, we detected cytoplasmic distribution of these fluorescent markers in fluidized RAB5A-expressing monolayers, indicative of NE ruptures (Fig. 3D-E and Movie S6).

Finally, we performed Correlative-Light Electron Microscopy (CLEM) tomography and immune EM to visualize directly the presence of NE ruptures. EGFP-cGAS control and RAB5A-expressing cells were processed for immunofluorescence to detect perinuclear foci of EGFP-cGAS and, subsequently, for EM tomography. Specimens were concomitantly stained with gold-labeled anti-EGFP antibodies to reveal cGAS, which accumulated at sites of condensed chromatin, immediately adjacent to the region where both the inner and outer NE membranes were ruptured (Fig. 3F and Fig. S5B). Interestingly, immunofluorescent staining of Lamin $\mathrm{A} / \mathrm{C}$ also showed that nuclei in RAB5A-fluidized monolayers undergo large deformation and possibly ruptures as indicated by the accumulation of cGAS around distorted nuclei and at the apex of nuclear invagination (Fig. S5C).

A chronic mechano-response underlies changes in nuclear rigidity, and heterochromatin structure, and perinuclear actin polymerization in RAB5A-fluidized collectives 
The large fluctuations in tissue density, cell, and nuclear shape suggest that RAB5A-fluidized epithelial collectives are subjected to persistent and chronic mechanical strain and stress. The accumulation of mechanical stress within tissues can compromise tissue integrity ${ }^{42}{ }^{43}$, particularly in cancerous cells, for which mechanical deformations have been shown to induce nuclear rupture and DNA damage ${ }^{33,34,44}$. Both individual cells and epithelial sheets, however, can adapt to acute short-lived stress by mounting a nuclear mechano-protective response that preserves them from widespread genomic damages ${ }^{45,46}$. Prolonged stretching or compressive mechanical stresses, however, frequently increases nuclear rigidity and size, elevation in chromatin compaction ${ }^{46-48}$, and the remodelling of peri-nuclear cytoskeletal actin with the formation of prominent nuclear actin rings ${ }^{49,50}$. We hypothesized that endocytic unjamming via flocking in epithelial ensembles exerts protracted mechanical stress, which instigates a protection strategy, which, however, fails eventually resulting in DNA damage. We set out to investigate this possibility.

Firstly, we investigated how nuclei respond to motility-induced fluctuation in the local cell density $\rho$, which were previously studied in both jammed and fluid monolayers ${ }^{12,51,52}$. To this end, we considered the dependence of the nuclear strain rate $\dot{\gamma}_{N}=\frac{1}{A} \frac{\partial A}{\partial t}$ on the local monolayer strain rate $\dot{\gamma}_{C}=-\frac{1}{\rho} \frac{\partial \rho}{\partial t}$, which we estimated as the divergence of the velocity field measured through PIV analysis (Fig. 4A-C). In all cases, a robustly close-to-linear, correlation is found between $\dot{\gamma}_{N}$ and $\dot{\gamma}_{C}$, indicating that the nucleus systematically deforms in response to compressive and tensile strains imposed on the cell by the relative motion of its neighbors. The ratio $\dot{\gamma}_{c} / \dot{\gamma}_{n}$ between the monolayer and the nuclear strain rate is, thus, an indicator of nuclear stiffness (see Methods for details). RAB5A-fluidized monolayers undergo larger density fluctuations (as captured by the RMS value of the divergence of the velocity field) compared to control-jammed monolayers (Fig. 4B-C). Furthermore, the linear relation between nuclear strain rate and monolayer strain rate is characterized by markedly different slopes in the two cases: in response to the same variation in the cell density, nuclei of RAB5A-fluidized monolayers deform less (by about a factor of 2), showing a markedly stiffer mechanical response compared to controls (Fig 4D-E).

Secondly, we showed that nuclear-projected areas are nearly $25 \%$ larger in RAB5A-fluidized monolayers as compared to control ones (Fig. S6A-B). Nuclei of RAB5A-expressing cells appeared isotropically and homogenously enlarged, and their nuclear envelope was under tension. These conditions have recently been shown to mediate the activation of adaptive mechanoresponses increasing the cPLA2mediated production of arachidonic acid and actomyosin contractility ${ }^{53-55}$. Noticeably, treatment with MK886 inhibits 5-lipoxygenase-activating protein (FLAP) and COX-1, thereby blocking the conversion of arachidonic acid into prostaglandins ${ }^{56}$ or with Blebbistatin, which impairs actomyosin contractility, partially reduced the RAB5A-dependent tissue fluidification-mediated upregulation of cytoDR (Fig. S6C).

Thirdly, to probe the heterochromatin state, we initially examined the nuclear levels of H3K27me3. RAB5A-fluidized DCIS cells display a small but significant increase in H3K27m3-heterochromatin marks (Fig. 4F-G), which were prominently enriched at the nuclear periphery (Fig. 4F-I). Analysis of the top 100 upregulated genes revealed among the top transcription factors, EZH2, a histone H3 Lysine $27 \mathrm{~N}-$ methyltransferase, and SUZ12, which is a key component of the Polycomb Repressor complex -2 (PRC2) (Fig. S6D). These enzymes deposit H3K27m3 to repress transcription and promote chromatin compaction in response to nuclear mechanical stress ${ }^{57}$. Additionally, a pre-ranked gene set enrichment analysis showed enrichment in genes that can be targeted by PRC2 (Fig. S6E). Consistently, silencing of EZH2 or SUZ12 abrogated the increase in H3K27me3-heterochromatin marks (Fig. 4G-H and Fig. S6F-G). Polycommediated heterochromatin compaction contributes to nuclear rigidity and nuclear resistance to deformation. It may also generate anchoring points for the LINC (Linker of Nucleoskeleton and Cytoskeleton) complex to strengthen the interaction between the cytoskeleton and the nucleus, thus making it easier for the cytoskeleton to move the nucleus within the cell ${ }^{58,59}$. Consistently, measurement of the mean square angular displacement $M S A D(\tau)$ revealed that nuclei in RAB5A-fluidized MCF10A or MCF10.DCIS.com monolayers displayed faster rotational dynamics, both in terms of the angular velocity $\omega$ associated with the observed short-time ballistic scaling $\operatorname{MSAD}(\tau) \sim(\omega \tau)^{2}$ and of the rotational diffusion coefficient $D_{R}$ capturing the long-time diffusive-like behavior $M S A D(\tau) \sim 2 D_{R} \tau$ (Fig. S7A). Additionally, while nuclei of control epithelial monolayers display a significant instantaneous correlation between nuclear orientation and direction of motion, there is an evident loss of correlation in RAB5A-fluidized cells, and specifically in MCF10.DCIS.com cells (Fig. S7B). Thus, RAB5A-fluidized collectives, display faster and more persistent rotational dynamics: all features that might contribute to exert increased nuclear mechanical strain.

The chronic mechanical stress together with Lamin B1 reduction in RAB5A-fluidized monolayers might also elicit genome-wide structural alterations in constitutive H3K9me3 normally associated with the lamina, as a mechanism to dissipate forces ${ }^{49}$. We employed Sammy-Seq and H3K9me3 CHIP-seq to verify this 
possibility. Sammy-Seq is a high-throughput sequencing-based method for genome-wide characterization of chromatin accessibility, which can detect architectural rearrangements of lamina-associated heterochromatin domains (LADs) ${ }^{60}$. RAB5-fluidized monolayers displayed no changes in the H3K9me3genome-wide ChIP-seq profile (Fig. S7C), but a consistent reduction in the SAMMY-seq signal for heterochromatin regions (Fig. S7D).

Next, we found that tissue fluidification is also accompanied by the perinuclear remodelling of the actin cytoskeleton. Inspection of cell and cytoskeletal architecture revealed drastic and time-dependent alterations in their organization in fluidized monolayers. Firstly, doxycycline induction of RAB5A resulted in perturbations of the shape of cells that switched from regular polygons in jammed monolayers to irregular and variably elongated shapes in fluidized collectives (Fig. 4J and Fig. S7E). This result agrees with the concept that cell shape elongation and variance in cell shape are strictly associated with unjamming and tissue fluidification ${ }^{61,62}$. This change of shape was accompanied by the presence of supracellular actin structures, by the increase in cytoplasmic polymerized actin, and by the formation of prominent perinuclear actin rings (Fig. 4J-K and Fig. S7E). As expected, interference with actin polymerization by the addition of latrunculin $B$ to sequester monomeric actin, inhibited the formation of these structures. More surprisingly, also CytoDR gene upregulation was found to be inhibited (Fig. S7F-G).

RAB5A-fluidized monolayers mount a complex mechano-protective response, which leads to decrease nuclear pliability and softness, suggesting the possibility that these monolayers are less capable of dissipating mechanical energy to prevent DNA damage ${ }^{49}$. Consistently, RAB5A-flocking monolayers display elevated DNA damage, as evidenced by the significant increase in 53BP1 and $\gamma \mathrm{H} 2 \mathrm{AX}$ foci (Fig. 5AC), and in the tail moment determined by neutral DNA comet assays ${ }^{63,64}$ (Fig. 5F-G).

\section{RAB5A-expression triggers cGAS activation and DNA damage in invasive ductal carcinoma in vivo and human patients}

Next, we studied whether the observed nuclear mechano-perturbations leading to cGAS activation, and increase in DNA damage observed in vitro are also relevant in pathological tissues. We first turned to orthotopic xenograft murine models of DCIS. Control and RAB5A-MCF10.DCIS.com cells were injected into the mammary fat pads of immunocompromised animals. In this system, RAB5A induction increased CytoDR, as expected (Fig. S8A). The immunohistochemical analysis further revealed that RAB5A tumors had elevated $\gamma \mathrm{H} 2 \mathrm{AX}$ positive cells (Fig. $6 \mathrm{~A}-\mathrm{B}$ ), and enhanced levels of cGAS, which display a perinuclear dotted or crescent-like appearance (Fig. 6C-D). This pattern is similar to the one seen in human DCIS (see Fig. S9C-D to compare the distribution of CGAS in human DCIS with local infiltrative areas), likely reflecting its activation by cytoplasmic DNA. The specificity of anti-cGAS antibodies was verified using FFPE MCF10.DCIS.com cells knocked down for cGAS (Fig. S8B-C).

Next, we focused on a set of human ductal carcinoma in situ with different degrees of local invasiveness. RAB5A expression was frequently elevated displaying a graded increase in expression at the margin of locally invasive foci of DCIS (Fig. 6E-G and S9A). In these regions, we have just shown that nuclei are significantly less rounded and display an increased number of cells expressing $\gamma \mathrm{H} 2 \mathrm{AX}$ as compared to the bulk of the tumor ${ }^{65}$. Multiplex Opal fluorescent staining revealed that marginal cells, which had elevated RAB5A, also displayed increased $\gamma \mathrm{H} 2 \mathrm{AX}$, and phosphorylated checkpoint kinase 1 (pChk1) (Fig. 6E-G and S9A), a marker of persistent DNA damage ${ }^{66}$, and more relevantly of cGAS (Fig. 6G-H and S9B). The latter protein was significantly more elevated in cells expressing high levels of RAB5A at the invasive foci of human DCIS, where it displayed either a perinuclear dotted or crescent-like appearance, similar to the one detected in RAB5A-expressing MCF10.DCIS.com orthotopic xenografts (Fig. S9C-D). Similar graded expression of RAB5A associated with elevated $\gamma \mathrm{H} 2 \mathrm{AX}$ and cGAS was also detected in patient-derived breast cancer organoids (Fig. S9E).

Quantitative analysis of a set of living breast cancer organoids labeled with NucLight to monitor nuclear motion revealed that some of them displayed persistent rotational motion and all the key features of a flocking fluid (Movie S7). By combining 3D image registration with a differential analysis of the residual intensity fluctuations (see Methods and Ref. ${ }^{67}$ for details), we decoupled the rigid body contribution associated with the global rotation of the organoid from the internal rearrangement dynamics, which is captured by the overlap parameter $Q(\tau)$. Persistently rotating organoids (characterized by an average angular speed larger than 0.03 cycles $/ h$, roughly corresponding to one full rotation per day) also displayed markedly faster internal dynamics, with a relaxation time $\tau^{*}$ at least two times shorter than the static ones (Fig. S10A-C and Movie S8). This latter discovery further supports the pathophysiological relevance of fluidification via flocking. Next, we subjected the two static and the three rotating organoids to a genomic analysis by RNA-seq. Despite the limited number of samples, we found that rotating fluid organoids display 
a significantly elevated expression of RAB5A (Fig. S10D), enrichment in several genes belonging to the interferon-alpha stimulated and Interferon related DNA damage resistance signature (Fig. S10E-H). Whereas the analysis of the top deregulated genes pointed to PRC2 complex components, SUZ12 and $\mathrm{EZH} 2$, as a key altered transcription factor in rotating organoids (Fig. S10I).

\section{Tissue fluidification via flocking enriches for plastic epithelial-to-mesenchymal traits and increases} chemoresistance

Chronic stimulation of CGAS-STING signaling has been shown to exert either immunoprotective or protumorigenic effects. For example, by establishing an immune-suppressive tumor microenvironment, cGAS activation can promote a transition toward a mesenchymal state ${ }^{68}$ and resistance to chemotherapeutic agents that result in increased metastasis ${ }^{69}$. In addition, an experimentally derived interferon-related DNA damage resistance signature (IRDS) highly related to CytoDR has been associated with resistance to chemotherapy and/or radiation across different cancer cell lines ${ }^{70}$. Hence, we hypothesize that endocyticmediated tissue fluidification might promote the acquisition of chemoresistance and plastic EMT traits.

Firstly, we noticed that several mesenchymal markers, including CDH2, ZEB1, MMP13, EGF, and AXIN2, were reproducibly and significantly upregulated in RAB5A-expressing fluidized-via-flocking MCF10.DCIS.com (Fig. 7A). Several canonical mesenchymal factors, including the master EMT regulators, SNAIL1 and 2 and TWIST, were unaffected (not shown), suggesting the acquisition of what has been defined as plastic EMT transcriptional state $(E M P)^{71}$. This was corroborated by the morphological analysis, which indicated that RAB5A induction leads to the loss of the typical cuboidal epithelial shape with the acquisition of an elongated mesenchymal morphology (Fig. 7B). Notably, the expression of this set of genes was dependent on the activity of YAP1/TAZ (Fig. S11A-B), consistent with the concept that the EMP phenotype switch is, at least in part, a mechanoresponsive process ${ }^{72-77}$.

Finally, we determined whether the elevation of CytoDR is associated with the acquisition of chemoresistance to anti-tumorigenic drugs. Firstly, by examining the differential gene expression profile of control and fluidized monolayers, we observed a concordant interferon-related DNA damage resistance signature that has been previously associated with resistance to chemotherapy and/or radiation particularly in breast cancer ${ }^{70}$ (Fig. S7C). Additionally, we found that RAB5A -expressing cells were slightly more resistant to the topoisomerase inhibitor, etoposide, and remarkably more resistant to docetaxel (Fig. 7DE). In this latter case, while most control cells display a grossly defective nuclear morphology in keeping with the antimitotic activity of taxols, more than $60 \%$ of RAB5A-expressing displayed intact and unperturbed nuclei (Fig. 7F).

\section{Conclusions and outlook}

The tissue-level phase transition from a solid or jammed to a liquid-like or unjammed state has been recently proposed to be a complementary or, possibly, alternative gateway to cell invasion in both normal epithelia during development ${ }^{3}$ and in solid carcinoma during malignant progression ${ }^{2,4,9,61}$. Experimental evidence indicated that the progression from an indolent, quasi benign ductal breast carcinoma lesion to invasive ductal carcinoma is associated with the acquisition of a flocking-fluid mode of collective motion induced by the upregulation of the endocytic, promigratory gene, RAB5A ${ }^{2}, 10$. Indeed, fluidized RAB5A-expressing DCIS spheroids display a radial gradient of fluidity with cells at the periphery in contact with the ECM exhibiting faster dynamics and increased tissue fluidization ${ }^{2}$. These rapid and dynamic changes impose mechanical strains and stresses on the surrounding ECM fibers, ultimately resulting in their remodeling and the generation of tracks and channels that facilitate collective invasion ${ }^{2}$.

Here, we showed that in addition to these short-time features, the altered mechanics of fluidized tissues is also accompanied by a robust, cell-autonomous, and long-lived transcriptional rewiring mediated by the cGAS/STING-dependent, proinflammatory response.

Previous studies showed that cancer cell invasion into the confinement of narrow channels and gaps of interstitial tissues exerts large mechanical strains on the nucleus that, when above a certain threshold, can lead to nuclear envelope ruptures (NER) and transient leakage of DNA into the cytosol33, 34, 44, 78. These events, however, are typically short-lived as NER are efficiently and rapidly repaired by the Endosomal Sorting Complex Required for Transport (ESCORT III) system ${ }^{33,79}$ and the Barrier-to-autointegration factor $(\mathrm{BAF})^{80}$, and have not been shown to promote permanent cell fate changes or transcriptional rewiring. BAF, specifically, can also inhibit cGAS binding to DNA and cGAS activation ${ }^{81}$. Tissue fluidification via flocking results, however, in a persistent and a long-lived, chronic mechanical stress state. Tumor cells react to these challenges by mounting a set of diverse mechanoprotective response strategies ${ }^{49}$, including 
increasing nuclear stiffening, alterations in the distribution and structures of heterochromatin, loss of Lamin $\mathrm{B} 1$, and the formation of persistent and nuclear protective actin rings ${ }^{49}$. However, as mechanical strains driven by large-scale tissue fluctuations and small-scale nuclear deformations persist in fluidized tissues, nuclear mechanoprotection eventually fails. This results in frequent and repeated NER, and in mechanically-induced, aberrantly elevated DNA damage. These combined events might lead to the accumulation of fragmented DNA that overcome the inhibitory action of BAF and potently activates sustained cGAS/STING signaling, leading to rewiring of the transcriptional profile of DCIS toward an innate immunity, interferon-like, inflammatory response. Remarkably, such changes are a cell-intrinsic and an emerging property of tumor cell collectives, rather than dependent on the interaction of the malignant tissue with the microenvironment. It must be noted, however, that mechanical perturbations associated with tissue fluidification via unjamming have recently been shown to be driven also by extreme ECM-mediated confinement during invasive growth of triple-negative mammary cancer ${ }^{82}$ or by compressive stresses ${ }^{83}$. These microenvironmental-driven alterations might also contribute to the activation of proinflammatory, transcriptional changes. Indeed, we showed that in naturally occurring DCIS the activation of cGAS and the increased elevation of $\gamma \mathrm{H} 2 \mathrm{AX}$ frequently accompany the formation of infiltrative foci that mark the transition toward a more invasive phenotype. At these sites, like in model DCIS in 2D and 3D, and in living breast cancer organoids, invasive buds display a graded elevation of RAB5A expression, associated with persistent angular motility and local gradients of fluidification. These local alterations in tissue dynamics are expected to increase chronic mechanical stress, thereby instigating conditions that facilitate NER and cGAS/STING elevation. Recently, we have also shown that extreme confinement in DCIS cancer model cells might be sufficient to promote NERs ${ }^{65}$. These events, however, are relatively transient, but sufficient to promote the nuclear entry of exonucleases, such as TREX1. TREX1 nuclear re-localization, in turn, critically contributes to damage the DNA, under conditions in which the leakage of DNA into the cytoplasm is limited and cGAS is not activated ${ }^{65}$.

Recently, skin epidermis stem/progenitor cells subjected to relatively short cycles of stretching have been shown to trigger amplitude-dependent supracellular and nuclear mechanoresponses ${ }^{49}$. These include the formation of transient actin rings and a nuclear tension-mediated, $\mathrm{Ca}^{++}$dependent reduction in the levels of $\mathrm{H} 3 \mathrm{~K} 9 \mathrm{me} 3$ heterochromatin, resulting in chromatin fluidification and nuclear softening to dissipate mechanical energy ${ }^{49}$. Sustained chromatin fluidification, in turn, led to transcriptional repression and decreased expression of cell identity and differentiation genes ${ }^{49}$. Unjamming via flocking imposes, however, qualitative and quantitative different mechanical strains, that, nevertheless, elicit a robust set of mechanoprotective responses. These protective strategies, like in skin epidermis stem/progenitor cells, include the permanent formation of actin rings, but no alterations in the global constitutive levels of H3K9me3 heterochromatin, which is, nevertheless, architecturally reorganized. In addition, we detected a slight global increase and a robust redistribution at the nuclear periphery of the facultative H3K27me3 marks. Notably, this latter modification has been shown to occur upon long-term (6-24 hours) stretching, and to be responsible for increase nuclear rigidity ${ }^{46,49}$, similar to what we found in fluidized normal and oncogenic mammary epithelia. Persistency of these alterations in the presence of constant mechanical stress exerted on cells and nuclei results in NER, leakage of cytoplasmic DNA, and DNA damage. Thus, cells mount distinctive mechanoprotective responses that are not only dependent on the time and extent of mechanical perturbations but are likely cell context related.

In the oncogenic DCIS setting, this mechanism of long-term adaptation to perturbed mechanics leads to the potent activation of the cGAS/STING axis. It is of note, that recently this cytosolic DNA response axis has been shown to induce a pro-tumorigenic phenotype, characterized by a shift toward a mesenchymal state and increased chemoresistance ${ }^{68,84}$, as we also found in our system. It is however likely that in an immune-proficient context, the same axis might trigger a pro-immunogenic, potentially anti-tumoral response. Whether this is the case is certainly a matter of future investigation. Similarly, it will be paramount to determine what are the factors or conditions that tune the mechanically-driven cGAS/STING activation toward either a pro-tumorigenic or pro-immunogenic fate. 


\section{References}

1. Northey, J.J., Przybyla, L. \& Weaver, V.M. Tissue Force Programs Cell Fate and Tumor Aggression. Cancer Discov 7, 1224-1237 (2017).

2. Palamidessi, A. et al. Unjamming overcomes kinetic and proliferation arrest in terminally differentiated cells and promotes collective motility of carcinoma. Nat Mater 18, 1252-1263 (2019).

3. Mongera, A. et al. A fluid-to-solid jamming transition underlies vertebrate body axis elongation. Nature 561, 401-405 (2018).

4. Oswald, L., Grosser, S., Smith, D.M. \& Kas, J.A. Jamming transitions in cancer. J Phys D Appl Phys 50, 483001 (2017).

5. Bi, D., Yang, X., Marchetti, M.C. \& Manning, M.L. Motility-Driven Glass and Jamming Transitions in Biological Tissues. Physical Review X 6, 021011 (2016).

6. Park, J.A. et al. Unjamming and cell shape in the asthmatic airway epithelium. Nat Mater 14, 10401048 (2015).

7. Sadati, M., Taheri Qazvini, N., Krishnan, R., Park, C.Y. \& Fredberg, J.J. Collective migration, and cell jamming. Differentiation 86, 121-125 (2013).

8. Malinverno, C. et al. Endocytic reawakening of motility in jammed epithelia. Nat Mater 16, 587-596 (2017).

9. Park, J.A., Atia, L., Mitchel, J.A., Fredberg, J.J. \& Butler, J.P. Collective migration and cell jamming in asthma, cancer, and development. J Cell Sci 129, 3375-3383 (2016).

10. Frittoli, E. et al. A RAB5/RAB4 recycling circuitry induces a proteolytic invasive program and promotes tumor dissemination. J Cell Biol 206, 307-328 (2014).

11. Giavazzi, F. et al. Flocking transitions in confluent tissues. Soft Matter 14, 3471-3477 (2018).

12. Giavazzi, F. et al. Giant fluctuations and structural effects in a flocking epithelium. Journal of Physics D: Applied Physics 50, 384003 (2017).

13. Cerbino, R. et al. Disentangling collective motion and local rearrangements in $2 \mathrm{D}$ and $3 \mathrm{D}$ cell assemblies. Soft Matter (2020).

14. Miller, F.R., Santner, S.J., Tait, L. \& Dawson, P.J. MCF10DCIS.com xenograft model of human comedo ductal carcinoma in situ. J Natl Cancer Inst 92, 1185-1186 (2000).

15. Hu, M. et al. Regulation of in situ to invasive breast carcinoma transition. Cancer cell 13, 394-406 (2008).

16. Hu, M.M. \& Shu, H.B. Innate Immune Response to Cytoplasmic DNA: Mechanisms and Diseases. Annu Rev Immunol 38, 79-98 (2020).

17. Kaur, H. et al. RNA-Seq of human breast ductal carcinoma in situ models reveals aldehyde dehydrogenase isoform 5A1 as a novel potential target. PLoS ONE 7, e50249 (2012).

18. Pulaski, B.A. \& Ostrand-Rosenberg, S. Mouse 4T1 breast tumor model. Curr Protoc Immunol Chapter 20, Unit 2022 (2001).

19. Lang, E. et al. Coordinated collective migration and asymmetric cell division in confluent human keratinocytes without wounding. Nat Commun 9, 3665 (2018).

20. Chen, Q., Sun, L. \& Chen, Z.J. Regulation and function of the cGAS-STING pathway of cytosolic DNA sensing. Nat Immunol 17, 1142-1149 (2016).

21. Gao, D. et al. Cyclic GMP-AMP synthase is an innate immune sensor of HIV and other retroviruses. Science 341, 903-906 (2013).

22. Lahaye, X. et al. The capsids of HIV-1 and HIV-2 determine immune detection of the viral cDNA by the innate sensor cGAS in dendritic cells. Immunity 39, 1132-1142 (2013).

23. Sun, L., Wu, J., Du, F., Chen, X. \& Chen, Z.J. Cyclic GMP-AMP synthase is a cytosolic DNA sensor that activates the type I interferon pathway. Science 339, 786-791 (2013).

24. Woo, S.R. et al. STING-dependent cytosolic DNA sensing mediates innate immune recognition of immunogenic tumors. Immunity 41, 830-842 (2014).

25. Rongvaux, A. et al. Apoptotic caspases prevent the induction of type I interferons by mitochondrial DNA. Cell 159, 1563-1577 (2014).

26. White, M.J. et al. Apoptotic caspases suppress mtDNA-induced STING-mediated type I IFN production. Cell 159, 1549-1562 (2014).

27. West, A.P. et al. Mitochondrial DNA stress primes the antiviral innate immune response. Nature 520, 553-557 (2015).

28. Gluck, S. et al. Innate immune sensing of cytosolic chromatin fragments through cGAS promotes senescence. Nat Cell Biol 19, 1061-1070 (2017). 
29. Harding, S.M. et al. Mitotic progression following DNA damage enables pattern recognition within micronuclei. Nature 548, 466-470 (2017).

30. Mackenzie, K.J. et al. cGAS surveillance of micronuclei links genome instability to innate immunity. Nature 548, 461-465 (2017).

31. Yang, Z., Maciejowski, J. \& de Lange, T. Nuclear Envelope Rupture Is Enhanced by Loss of p53 or Rb. Mol Cancer Res 15, 1579-1586 (2017).

32. Clark, K. et al. Novel cross-talk within the IKK family controls innate immunity. Biochem J 434, 93104 (2011).

33. Raab, M. et al. ESCRT III repairs nuclear envelope ruptures during cell migration to limit DNA damage and cell death. Science 352, 359-362 (2016).

34. Denais, C.M. et al. Nuclear envelope rupture and repair during cancer cell migration. Science 352, 353-358 (2016).

35. Ahmed, B. et al. Mitochondrial and Chromosomal Damage Induced by Oxidative Stress in $\mathrm{Zn}(2+)$ Ions, ZnO-Bulk and ZnO-NPs treated Allium cepa roots. Sci Rep 7, 40685 (2017).

36. Nagayama, K. \& Fukuei, T. Cyclic stretch-induced mechanical stress to the cell nucleus inhibits ultraviolet radiation-induced DNA damage. Biomech Model Mechanobiol 19, 493-504 (2020).

37. Shah, P., Wolf, K. \& Lammerding, J. Bursting the Bubble - Nuclear Envelope Rupture as a Path to Genomic Instability? Trends Cell Biol 27, 546-555 (2017).

38. Dupont, S. et al. Role of YAP/TAZ in mechanotransduction. Nature 474, 179-183 (2011).

39. Wang, H. et al. cGAS is essential for the antitumor effect of immune checkpoint blockade. Proc Natl Acad Sci U S A 114, 1637-1642 (2017).

40. Gao, P. et al. Cyclic $\left[G\left(2^{\prime}, 5^{\prime}\right) \mathrm{pA}\left(3^{\prime}, 5^{\prime}\right) \mathrm{p}\right]$ is the metazoan second messenger produced by DNAactivated cyclic GMP-AMP synthase. Cell 153, 1094-1107 (2013).

41. Gentili, M. et al. The N-Terminal Domain of cGAS Determines Preferential Association with Centromeric DNA and Innate Immune Activation in the Nucleus. Cell Rep 26, 2377-2393 e2313 (2019).

42. Casares, L. et al. Hydraulic fracture during epithelial stretching. Nat Mater 14, 343-351 (2015).

43. Harris, A.R. et al. Characterizing the mechanics of cultured cell monolayers. Proc Natl Acad Sci U $S$ A 109, 16449-16454 (2012).

44. Xia, Y. et al. Nuclear rupture at sites of high curvature compromises retention of DNA repair factors. J Cell Biol 217, 3796-3808 (2018).

45. Latorre, M. \& Humphrey, J.D. Modeling mechano-driven and immuno-mediated aortic maladaptation in hypertension. Biomech Model Mechanobiol 17, 1497-1511 (2018).

46. Le, H.Q. et al. Mechanical regulation of transcription controls Polycomb-mediated gene silencing during lineage commitment. Nat Cell Biol 18, 864-875 (2016).

47. Damodaran, K. et al. Compressive force induces reversible chromatin condensation and cell geometry-dependent transcriptional response. Mol Biol Cell 29, 3039-3051 (2018).

48. Bonnevie, E.D. et al. Cell morphology and mechanosensing can be decoupled in fibrous microenvironments and identified using artificial neural networks. Sci Rep 11, 5950 (2021).

49. Nava, M.M. et al. Heterochromatin-Driven Nuclear Softening Protects the Genome against Mechanical Stress-Induced Damage. Cell 181, 800-817 e822 (2020).

50. Shao, X., Li, Q., Mogilner, A., Bershadsky, A.D. \& Shivashankar, G.V. Mechanical stimulation induces formin-dependent assembly of a perinuclear actin rim. Proc Natl Acad Sci U S A 112, E2595-2601 (2015).

51. Zehnder, S.M. et al. Multicellular density fluctuations in epithelial monolayers. Phys Rev E Stat Nonlin Soft Matter Phys 92, 032729 (2015).

52. Zehnder, S.M., Suaris, M., Bellaire, M.M. \& Angelini, T.E. Cell Volume Fluctuations in MDCK Monolayers. Biophys J 108, 247-250 (2015).

53. Venturini, V. et al. The nucleus measures shape changes for cellular proprioception to control dynamic cell behavior. Science 370 (2020).

54. Lomakin, A.J. et al. The nucleus acts as a ruler tailoring cell responses to spatial constraints. Science 370 (2020).

55. Enyedi, B., Jelcic, M. \& Niethammer, P. The Cell Nucleus Serves as a Mechanotransducer of Tissue Damage-Induced Inflammation. Cell 165, 1160-1170 (2016).

56. Koeberle, A. et al. MK-886, an inhibitor of the 5-lipoxygenase-activating protein, inhibits cyclooxygenase-1 activity and suppresses platelet aggregation. Eur $J$ Pharmacol 608, 84-90 (2009). 
57. Heo, S.J. et al. Differentiation alters stem cell nuclear architecture, mechanics, and mechanosensitivity. Elife 5 (2016).

58. Liu, L. et al. Chromatin organization regulated by EZH2-mediated H3K27me3 is required for OPNinduced migration of bone marrow-derived mesenchymal stem cells. Int J Biochem Cell Biol 96, 29-39 (2018).

59. Gerlitz, G. \& Bustin, M. The role of chromatin structure in cell migration. Trends Cell Biol 21, 6-11 (2011).

60. Sebestyen, E. et al. SAMMY-seq reveals early alteration of heterochromatin and deregulation of bivalent genes in Hutchinson-Gilford Progeria Syndrome. Nat Commun 11, 6274 (2020).

61. Grosser, S. et al. Cell and Nucleus Shape as an Indicator of Tissue Fluidity in Carcinoma. Physical Review X 11, 011033 (2021).

62. Atia, L. et al. Geometric constraints during epithelial jamming. Nat Phys 14, $613-620$ (2018).

63. Wojewodzka, M., Buraczewska, I. \& Kruszewski, M. A modified neutral comet assay: elimination of lysis at high temperature and validation of the assay with anti-single-stranded DNA antibody. Mutat Res 518, 9-20 (2002).

64. Van Kooij, R.J. et al. The neutral comet assay detects double-strand DNA damage in selected and unselected human spermatozoa of normospermic donors. Int J Androl 27, 140-146 (2004).

65. Nader, G.P.F. et al. Compromised nuclear envelope integrity drives tumor cell invasion. bioRxiv, 2020.2005.2022.110122 (2020).

66. Niziolek-Kierecka, M., Dreij, K., Lundstedt, S. \& Stenius, U. gammaH2AX, pChk1, and Wip1 as potential markers of persistent DNA damage derived from dibenzo[a,I]pyrene and PAH-containing extracts from contaminated soils. Chem Res Toxicol 25, 862-872 (2012).

67. Cerbino, R. et al. Disentangling collective motion and local rearrangements in 2D and 3D cell assemblies. Soft Matter 17, 3550-3559 (2021).

68. Bakhoum, S.F. et al. Chromosomal instability drives metastasis through a cytosolic DNA response. Nature 553, 467-472 (2018).

69. Coussens, L.M. \& Werb, Z. Inflammation and cancer. Nature 420, 860-867 (2002).

70. Weichselbaum, R.R. et al. An interferon-related gene signature for DNA damage resistance is a predictive marker for chemotherapy and radiation for breast cancer. Proc Natl Acad Sci U S A 105, 18490-18495 (2008).

71. Yang, J. et al. Guidelines and definitions for research on epithelial-mesenchymal transition. Nat Rev Mol Cell Biol 21, 341-352 (2020).

72. Wei, S.C. et al. Matrix stiffness drives epithelial-mesenchymal transition and tumour metastasis through a TWIST1-G3BP2 mechanotransduction pathway. Nat Cell Biol 17, 678-688 (2015).

73. Kim, H.Y., Jackson, T.R. \& Davidson, L.A. On the role of mechanics in driving mesenchymal-toepithelial transitions. Semin Cell Dev Biol 67, 113-122 (2017).

74. Scott, L.E., Weinberg, S.H. \& Lemmon, C.A. Mechanochemical Signaling of the Extracellular Matrix in Epithelial-Mesenchymal Transition. Front Cell Dev Biol 7, 135 (2019).

75. Yuan, Y. et al. YAP overexpression promote the epithelial-mesenchymal transition and chemoresistance in pancreatic cancer cells. Mol Med Rep 13, 237-242 (2016).

76. Shao, D.D. et al. KRAS and YAP1 converge to regulate EMT and tumor survival. Cell 158, 171184 (2014).

77. Zhang, J. et al. YAP-dependent induction of amphiregulin identifies a non-cell-autonomous component of the Hippo pathway. Nat Cell Biol 11, 1444-1450 (2009).

78. Pfeifer, C.R. et al. Constricted migration increases DNA damage and independently represses cell cycle. Mol Biol Cell 29, 1948-1962 (2018).

79. Radulovic, M. \& Stenmark, H. ESCRTs in membrane sealing. Biochem Soc Trans 46, 773-778 (2018).

80. Halfmann, C.T. et al. Repair of nuclear ruptures requires barrier-to-autointegration factor. $J$ Cell Biol 218, 2136-2149 (2019).

81. Guey, B. et al. BAF restrict cGAS on nuclear DNA to prevent innate immune activation. Science 369, 823-828 (2020).

82. Ilina, O. et al. Cell-cell adhesion and 3D matrix confinement determine jamming transitions in breast cancer invasion. Nat Cell Biol 22, 1103-1115 (2020).

83. Kilic, A. et al. Mechanical forces induce an asthma gene signature in healthy airway epithelial cells. Sci Rep 10, 966 (2020). 
84. Vashi, N. \& Bakhoum, S.F. The Evolution of STING Signaling and Its Involvement in Cancer. Trends Biochem Sci (2021).

\section{Acknowledgments}

We thank Mirko Riboni, Simone Minardi, Valentina Dall'Olio, and Laura Tizzoni at Cogentech for sequencing and qRT-PCR services, Dario Parazzoli and the imaging unit of IFOM, IFOM cell culture facility for technical assistance

This work was supported by: Associazione Italiana per la Ricerca sul Cancro (AIRC IG\#18621 and 5Xmille\#22759 to GS, M.F., and C.T.; AIRC-IG-\#21416 to M.F.

MyFirstAIRCgrant MFAG\#22083 to FG; AIRC-IG \#21762 and AIRC 5X1000\#21091)

to F.d.F); the Italian Ministry of University and Scientific Research (PRIN 2017, 2017HWTP2K to GS; PRIN2015SJLMB9 to M.F. and F.d.F); the European Research Council (ERC advanced grant\#835103 to F.d.F); Progetto AriSLA 2021 "DDR \& ALS; FRRB - Fondazione Regionale per la Ricerca Biomedica - under the frame of EJP RD, the European Joint Programme on Rare Diseases with funding from the European Union's Horizon 2020 research and innovation program under the EJP RD COFUND-EJP N ${ }^{\circ} 825575$. to F.g.F; from LABEX DCBIOL (ANR-10-IDEX-0001-02 PSL* and ANR-11-LABX-0043), ANR ANR-17-CE150025-01 and ANR-18-CE92-0022-01, INSERM 19CS007-00, INCA PLBIO to N.M; INCA PLBIO 2019-1-PL BIO-07-ICR-1, INSERM Plan Cancer Single Cell grant 19CS007-00 to M.P.

H.A. is supported by a fellowship from Fondazione Umberto Veronesi; L.B, from a fellowship from the Associazione Italiana per la Ricerca sul Cancro.

\section{Author contributions:}

Conceptualization: GS, CT, FG, RC,

Methodology: EF, AP, SV, LB, HA, CM, GVB, GdC, FP, UG, VC, GN.

Data Analysis and quantification: FI, FZ, FG, SV, RC, WY, EM, CP, FF, CL

Visualization: AP, EF

Clinical sample acquisition, VG, GB, CT, VC, CR, ML, RC, PP, AV

Funding acquisition: SJE, MJM, JLS, EH

Supervision: MF, MP, FdF, MP, AM, VG, GB, FF, CL

Writing - original draft: GS, AP

Writing - review \& editing: GS, FG, RC, CT

Competing interests: M.P. is co-founder, member of the board of directors, and stakeholder of the company CheckmAb s.r.l. The remaining authors declare no competing interests.

Data and materials availability: RNA-seq data of MCF10A and MCF10.DCIS.com cells and organoids are being submitted respectively to Gene Expression Omnibus (GEO) and European Genome-phenome Archive (EGA) and are available upon request.

All other data are available in the main text or supplementary materials. 
Manuscript: Frittoli\&Palamidessi

August 2021

A

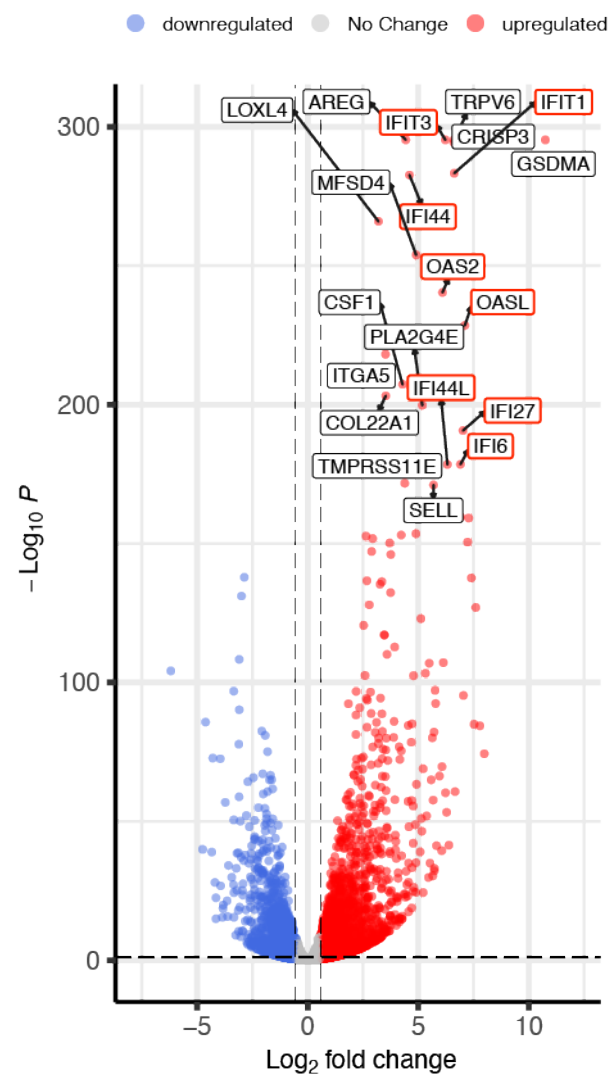

C

Cytosolic DNA sensing pathway

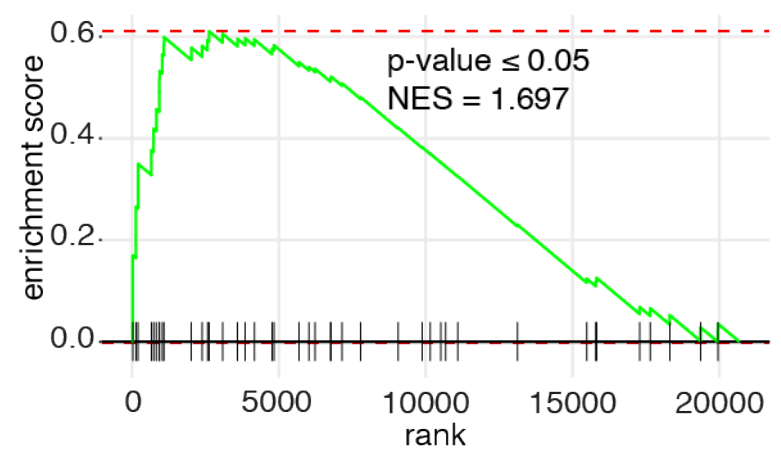

E

160 CTR RAB5A

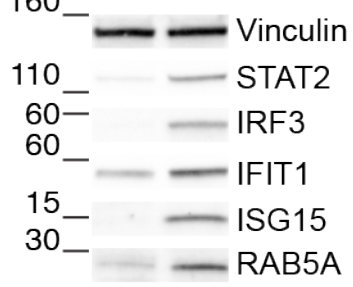

B

Figure 1

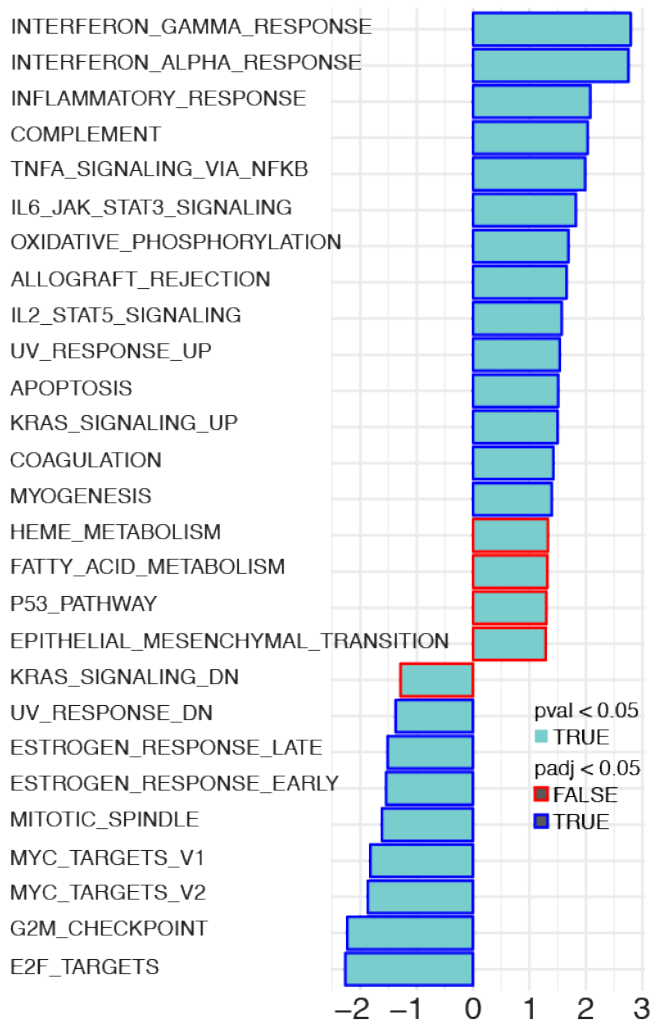

Normalized Enrichment Score

D

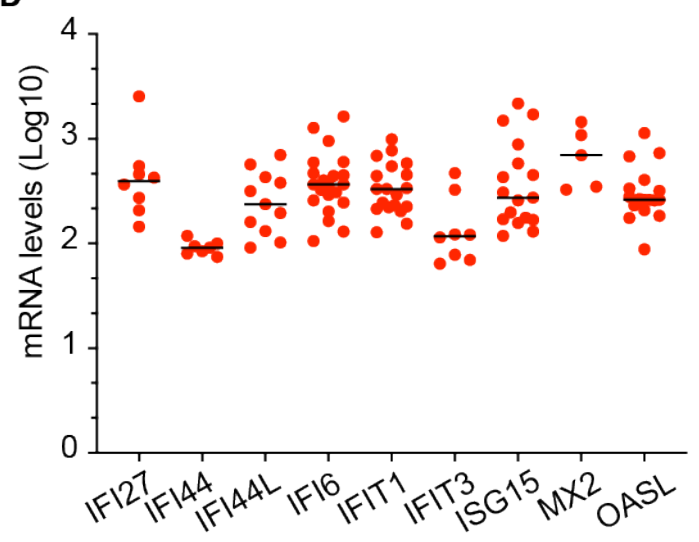




\section{Figure 1. Tissue fluidification induces a CytoDR gene signature}

A. Volcano plot representing differentially expressed genes between control empty vector and RAB5Aexpressing MCF10.DCIS monolayers. All significantly RAB5A-expressing deregulated genes are indicated in red (upregulated) and blue (downregulated). The enrichment (log2Fold Change) is plotted on the x-axis and the significance (Wald test -log10 p-value) is plotted on the y-axis. Labels are the most upregulated genes. Outlined in red are interferon-stimulated genes.

B. Gene set enrichment analysis (GSEA) of differentially expressed genes in RAB5A-expressing MCF10.DCIS.com monolayer over control cells. GSEA was performed using the Hallmarks pathway gene sets in the GSEA Molecular Signatures Database. Moderated t-statistic was used to rank the genes. Reported are significantly enriched pathways (P-value $<0.05)$ with the color of the outline of the bar corresponding to the $\mathrm{BH}$-adjusted $\mathrm{P}$-value. $\mathrm{P}$-value was calculated as the number of random genes with the same or more extreme ES value divided by the total number of generated gene sets.

C. GSEA Enrichment plot of differentially expressed genes in RAB5A-expressing MCF10.DCIS.com monolayer using the KEGG cytosolic DNA sensing pathway (hsa04623). The green curve corresponds to the ES (enrichment score) curve, which is the running sum of the weighted enrichment score obtained from GSEA software, while the normalized enrichment score (NES) and the corresponding P-value are reported within the graph.

D. Scatter plots of mRNA expression levels of IFI27, IFI44, IFI44L, IFI6, IFIT1, IFIT3, ISG15, MX2, and OASL determined by qRT-PCR in RAB5A-expressing MCF10.DCIS monolayers relative to control cells. Data are expressed as $\log _{10}$ values, horizontal lines represent group medians. Each dot represents an independent experiment. Values were normalized to the controls of each experiment

E. Immunoblots of lysates from doxycycline-treated control (CTR) and RAB5A-expressing (RAB5A) MCF10.DCIS.com monolayers with the indicated antibodies. Mw is indicated on the left. 
A

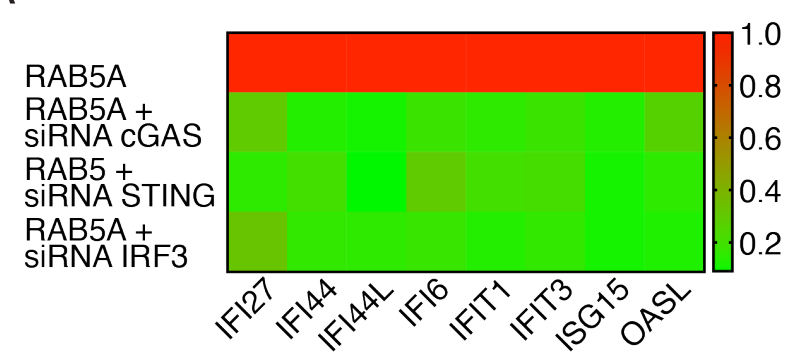

C

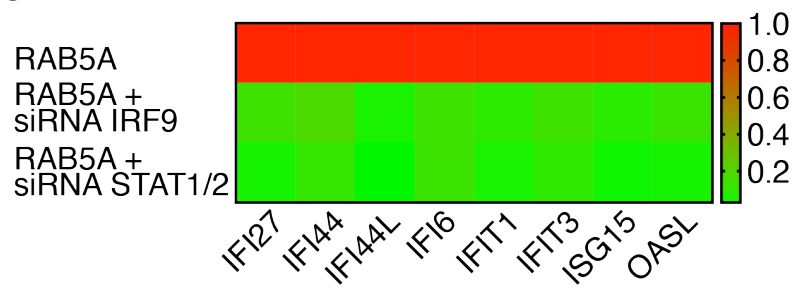

E

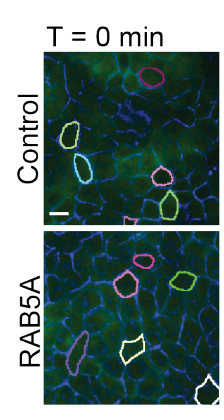

H

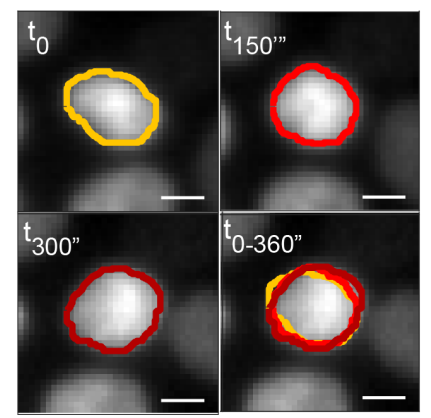

J
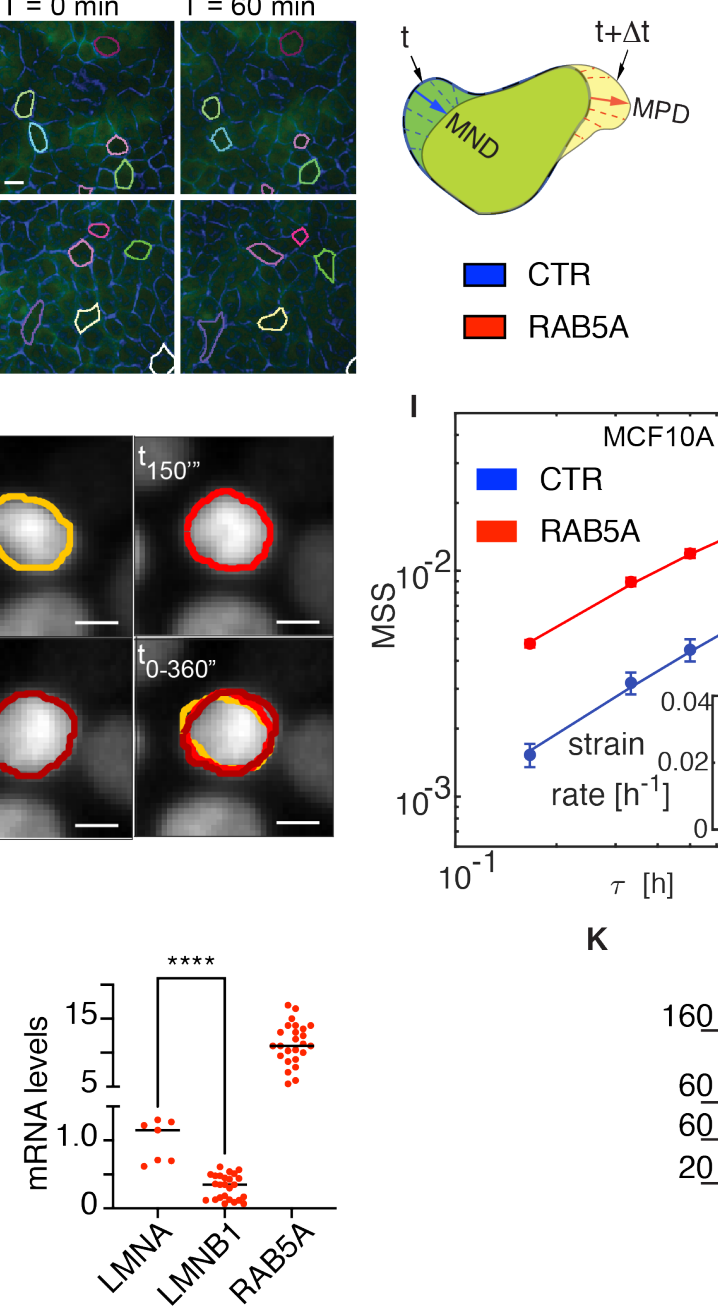

B

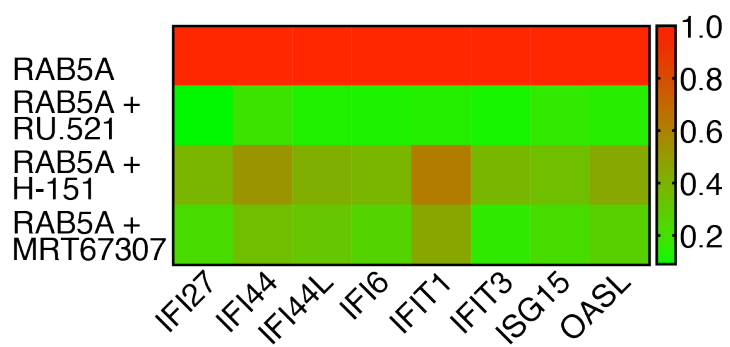

D

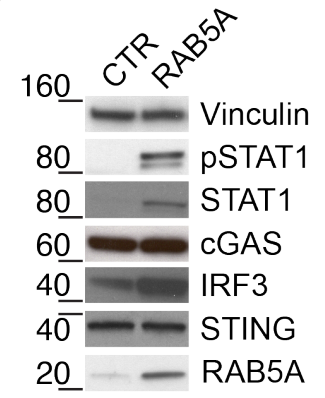

F G

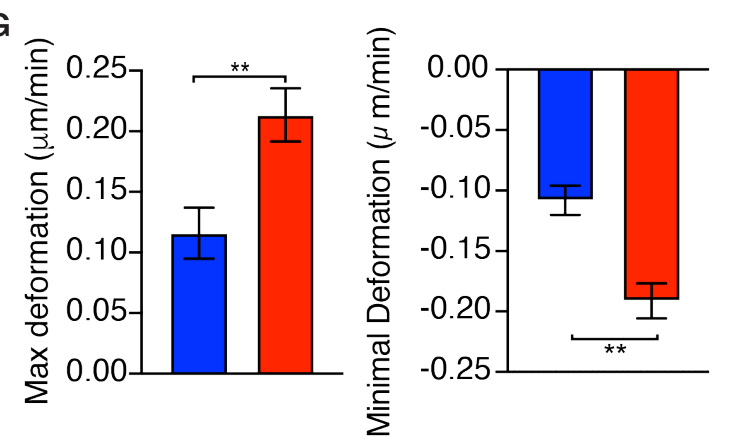
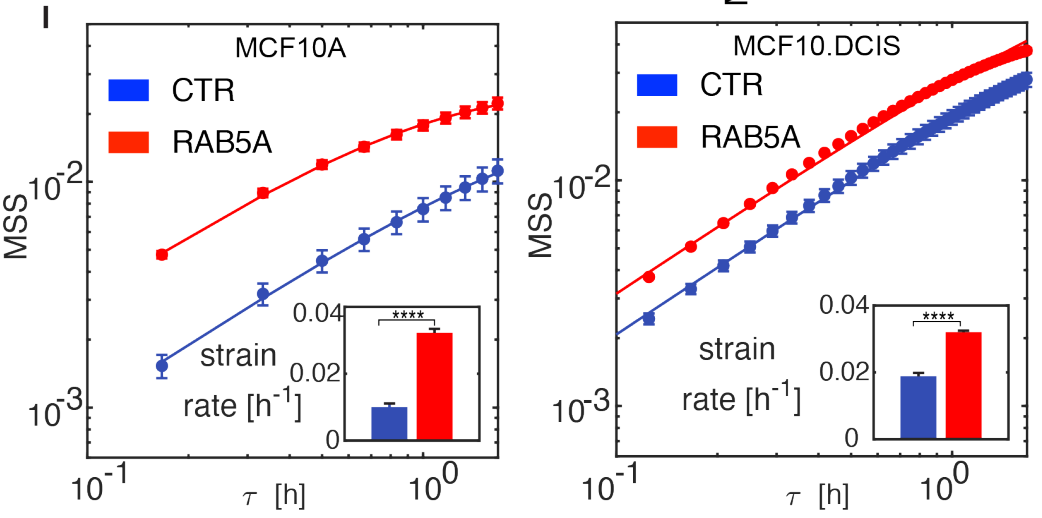

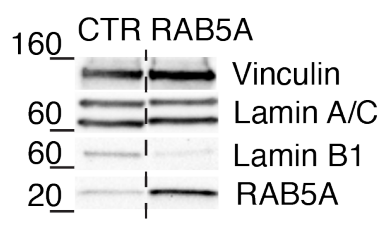




\section{Figure 2. A cGAS/STING pathway mediates tissue fluidification-dependent CytoDR genes}

A. Heatmap representing color-coded expression levels of differentially expressed CytoDR genes in RAB5A-expressing MCF10.DCIS.com monolayers silenced for the indicated genes. The effectiveness of silencing was determined by qRT-PCR (see also Figure S4A). The data are the ratio between the level of gene expression in each of the conditions tested relative to those of mock-treated (using scramble oligos) RAB5A-expressing cells. The mean \pm s.d. (at least $n=3$ independent experiments) and $P$ values, each-pair Student's t-test (treated versus mock-treated RAB5A-expressing cells) are reported in Table S1_Figure 2A. B. Heatmap representing color-coded expression levels of differentially expressed CytoDR genes in RAB5A-expressing MCF10.DCIS.com monolayers treated with the indicated small molecules: cGAS inhibitor, RU.521, the STING antagonist, H-151, or the TBK1/IKK inhibitor, MRT67307. The data are the ratio between the level of gene expression in each of the conditions tested relative to vehicle-treated RAB5A-expressing cells. The mean \pm s.d. (at least $n=3$ independent experiments) and $P$ values, each-pair Student's t-test (treated versus untreated RAB5A-expressing cells), are reported in Table S2_Figure 2B. C. Heatmap representing color-coded expression levels of differentially expressed Cyto $\bar{D} R$ genes in RAB5A-expressing MCF10.DCIS.com monolayers silenced for the indicated genes. The effectiveness of silencing was determined by qRT-PCR. The data are the ratio between the level of gene expression in each of the conditions tested relative to those of mock-treated (using scramble oligos) RAB5A-expressing cells. The mean \pm s.d. (at least $n=7$ independent experiments) and $P$ values, each-pair Student's t-test (siRNA versus RAB5A-expressing cells) are reported in Table S3_Figure 2C.

D. Immunoblots of lysates from doxycycline-treated control (CTR) and RAB5A-expressing (RAB5A) MCF10.DCIS.com monolayers with the indicated antibodies. Mw is indicated on the left.

E-G. Area fluctuations induced by RAB5A. EGFP-CDH1, control (CTR) and RAB5A-expressing (RAB5A) MCF10A monolayers seeded at a jamming density, were treated with doxycycline for $16 \mathrm{~h}$ and monitored by time-lapse over $24 \mathrm{~h}$ (5 min frame-rate) (Movie S3). Automated image segmentation was applied to identify the cell contour and area exploiting the EFGP-CDH1 fluorescence as described in methods. (E.) Still images depicting representative examples of selected cells with their contour indicated by pseudocoloring and used for the analysis are shown a $t=0$ and $t=60 \mathrm{~min}$. Scale Bar is $10 \mu \mathrm{m}$. (F.). Scheme depicting how the maximum positive deformation (MPD) and maximum negative deformation (MND) were scored and computed for each cell analyzed. (G.) Data are the mean of maximal and minimal area deformation over time. ( $n=80$ cells/conditions in one representative experiment out of four). ${ }^{* *} p<0.005$, Mann-Whitney paired-t-test

H. Nuclear segmentation. Representative consecutive frames centered on the same RAB5A-expressing MCF10A nucleus. Continuous lines with different shades of red represent the corresponding profiles obtained via nuclear segmentations. In the bottom right panel, the superposition of the three profiles is also shown, illustrating the characteristic shape variations the nucleus undergoes. Scale bar, $5 \mu \mathrm{m}$

I. Comparison of the nuclear mean square strain (MSS) of control and RAB5A-expressing cells in MCF10A (left panel) and MCF10.DCIS.com (right panel) monolayers. In each condition, the MSS is obtained by tracking and segmenting $N$ nuclei over the time window 4-20 h $(N>5000$ and $N>1000$ for control and RAB5A-expressing MCF10A monolayers, respectively; $N>700$ and $N>400$ for control and RAB5Aexpressing MCF10.DCIS.com monolayers, respectively). Continuous lines are best fitting curves to the data with an exponential model. The initial slope of each curve enables estimating the corresponding nuclear strain rate, which is reported in the inset of each panel as mean \pm s.d. ( $n=10$ randomly populated subsets of cells). ${ }^{* * *} p<0.0001, P$ values, T-test (CTR versus RAB5A).

J. Scatter plot of the mRNA expression levels of LMNA, LMNB1, and RAB5A determined by qRT-PCR in RAB5A-expressing MCF10.DCIS.com over control monolayers. The data are the mean (at least $n=7$ independent experiments). ${ }^{* * *} p<0.0001, P$ values, each-pair Mann Whitney test (LMNA versus LMNB1). K. Immunoblots of doxycycline-treated control (CTR) and RAB5A-expressing (RAB5A) MCF10.DCIS.com monolayers with the indicated antibodies. Mw is indicated on the left. 
A

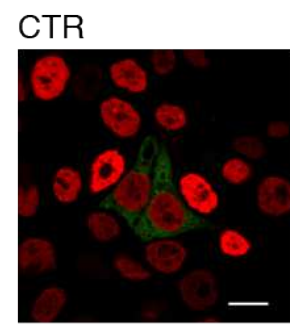

cherryH2B

GFP-CGAS

D

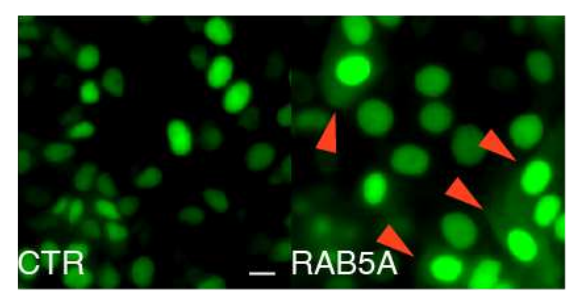

GFP-3NLS

F

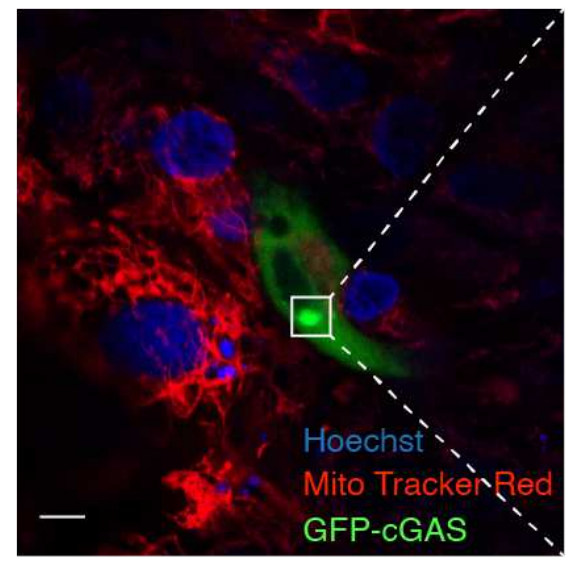

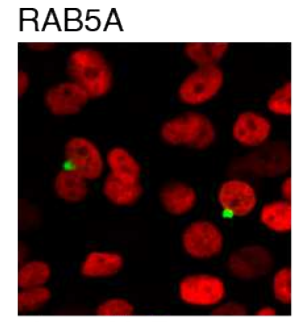

cherryH2B

GFP-cGAS
B

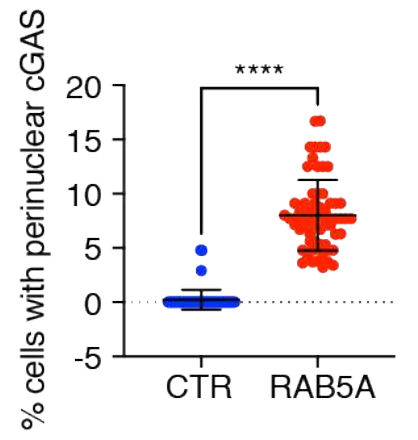

C

Figure 3

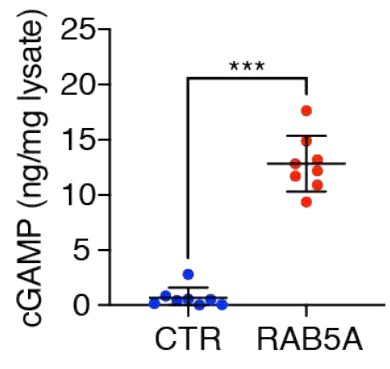

E
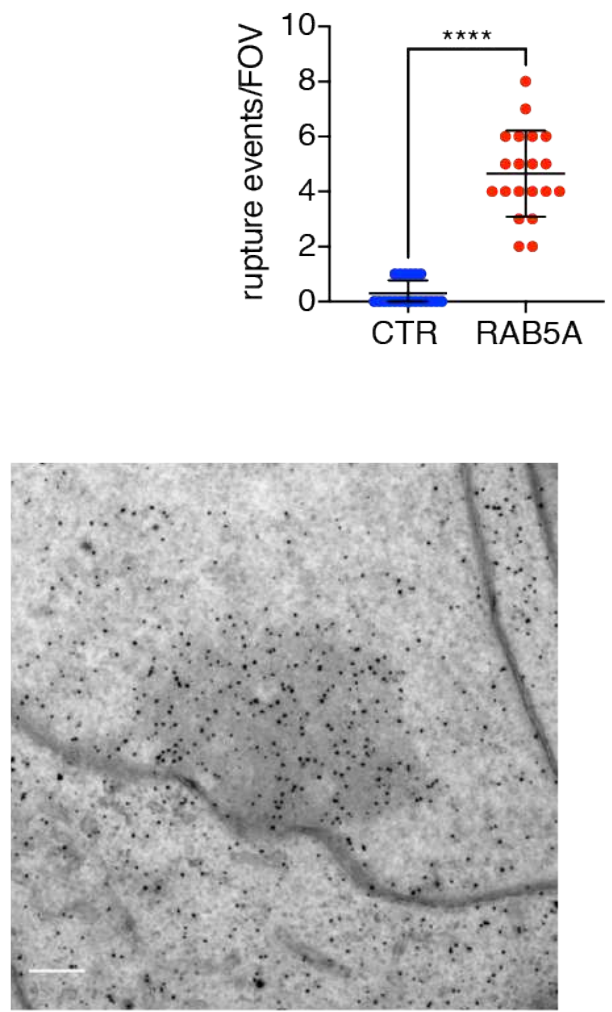

3D Tomography
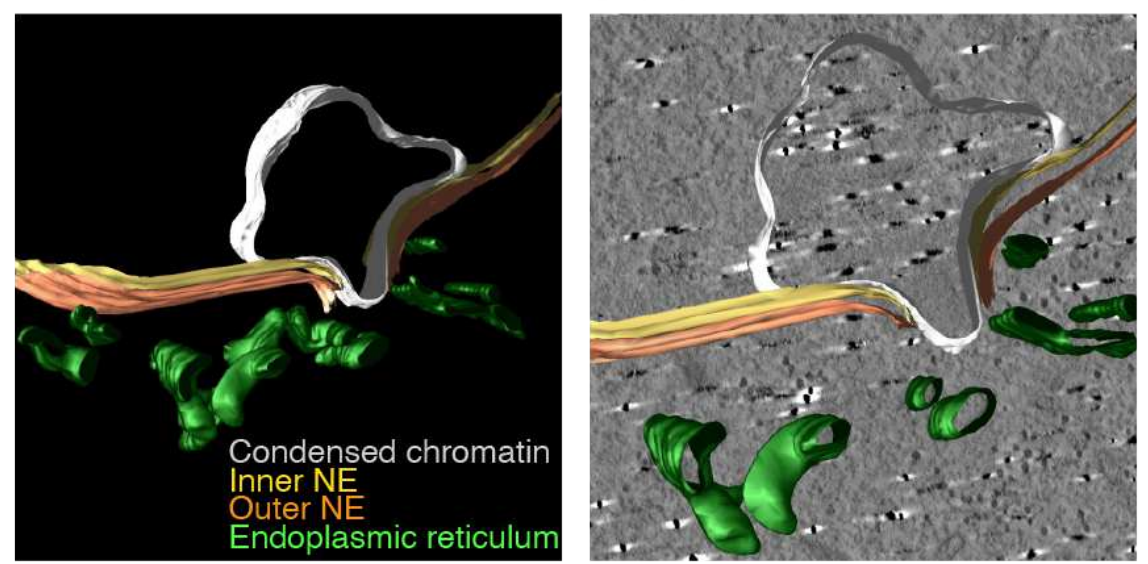
Figure 3. Nuclear envelope ruptures activate cGAS in fluidized monolayers

A. Immunofluorescence images of doxycycline-treated control (CTR) and RAB5A-expressing (RAB5A) mcherry-H2B MCF10.DCIS.com monolayers transfected with EGFP-cGAS. Scale bar $20 \mu \mathrm{m}$.

B. Scatter plot of the percentage of MCF10.DCIS.com cells with perinuclear cGAS enrichment is expressed as the mean \pm s.d. (at least $n=10 \mathrm{FOV} /$ experimental conditions in 3 independent experiments). ${ }^{* * * *} p<0.0001, P$ values, each-pair Mann Whitney test (CTR versus RAB5A).

C. Scatter plot of the quantification of cGAMP by ELISA from control (CTR) and RAB5A-expressing (RAB5A) MCF10.DCIS.com cell extracts. Data are the cGAMP amounts (ng) per mg of total cell extract expressed as the mean \pm s.d. (at least $n=3$ independent experiments). ${ }^{* \star *} p<0.0001, \mathrm{P}$ values, each-pair Welch's test (CTR versus RAB5A).

D. Snapshot of time-lapse (Movie S6) EGFP-3NLS-expressing control (CTR) and RAB5AMCF10.DCIS.com monolayers displaying events of NE rupture and EGFP-3NLS leakage (red harrowheads). Scale bar $10 \mu \mathrm{m}$.

E. Scatter plot of the number of nuclear envelope rupture events/FOV reported as mean \pm s.d. $(n=10$ FOV/experimental conditions in 2 independent experiments). ${ }^{* * *} p<0.0001, P$ values, each-pair Mann Whitney test (CTR versus RAB5A).

F. CLEM analysis of cGAS perinuclear foci. RAB5A-expressing MCF10.DCIS.com cells monolayers transfected with EGFP-cGAS were plated on MaTek dishes with grid and stained with Hoechst and Mito Tracker Red to detect nuclei and mitochondria respectively (upper left). Scale bar $5 \mu \mathrm{m}$. Cells identified on grids by confocal microscopy were processed for electron microscopy and z-axis serial sections were stained with gold-labeled anti-GFP antibody to detect EGFP-cGAS (upper right). Scale bar $500 \mathrm{~nm}$. Images of three-dimensional tomographic reconstruction and $3 \mathrm{D}$ model of a nuclear envelope rupture site (bottom panels). 
Manuscript: Frittoli\&Palamidessi

August 2021

772 
Figure 4

A

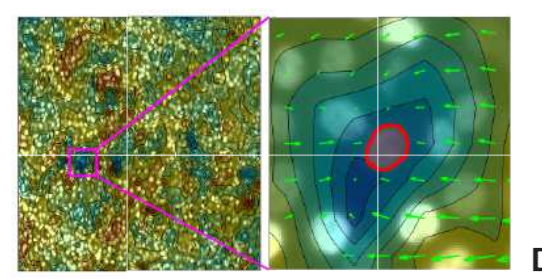

B

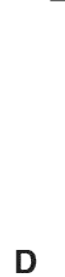

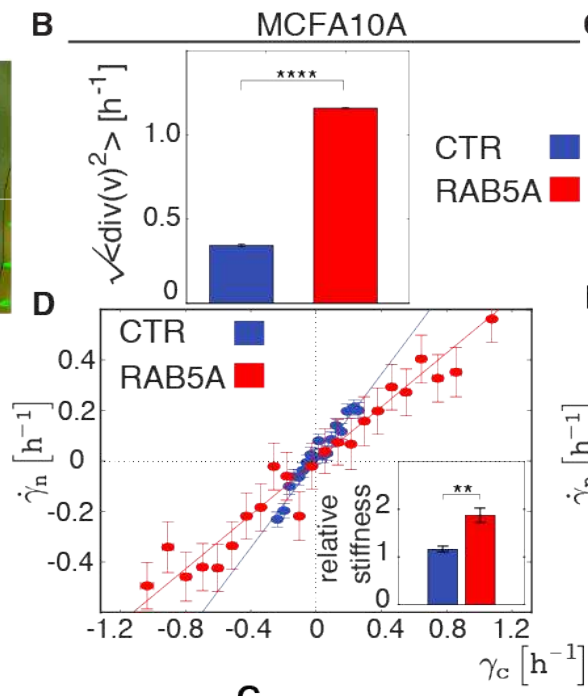
C MCFA10.DCIS
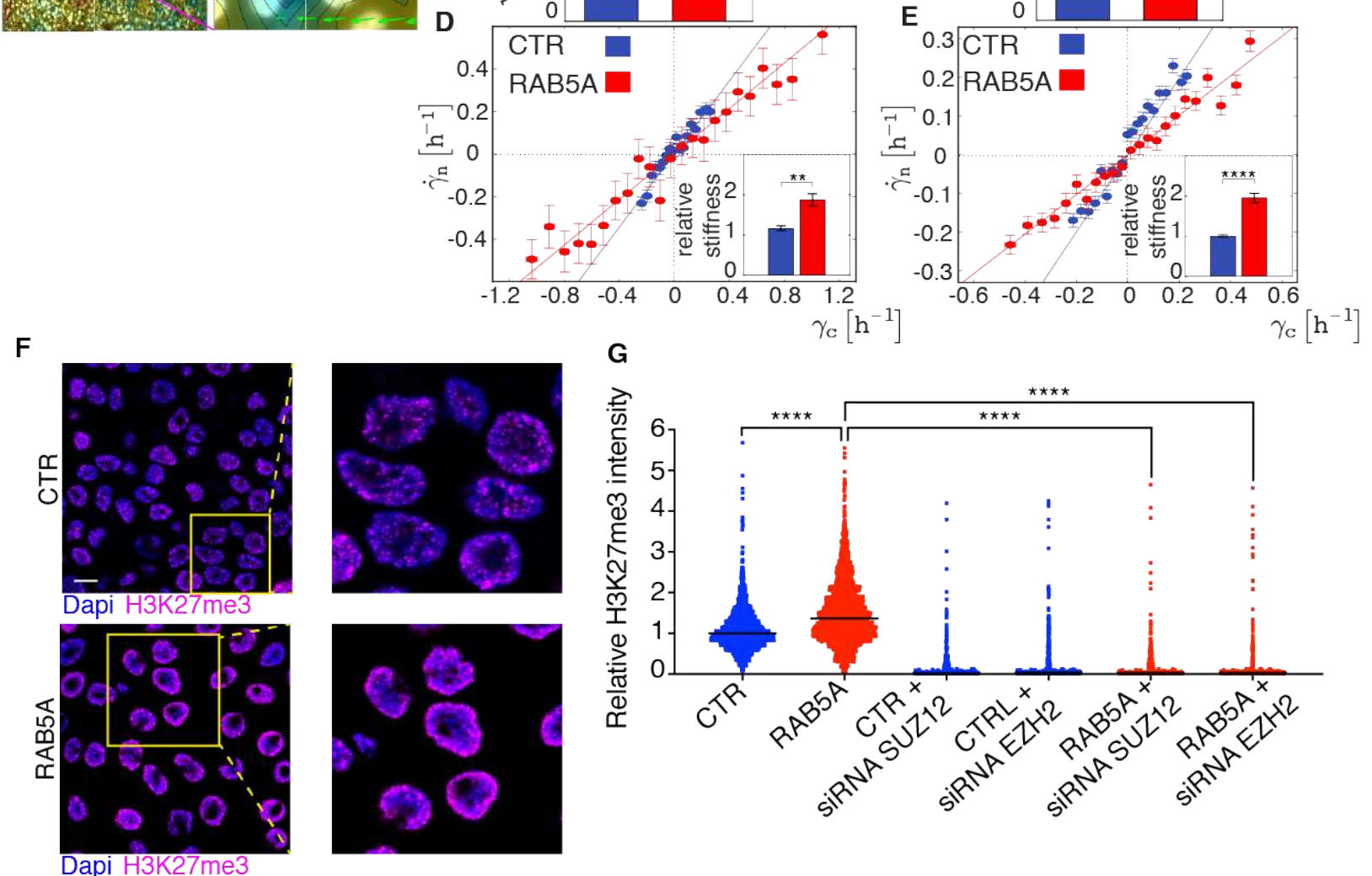

H

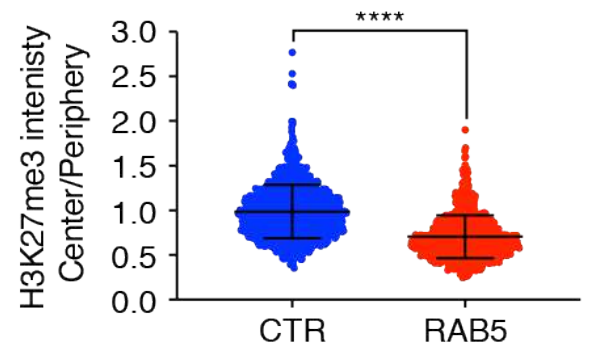

J

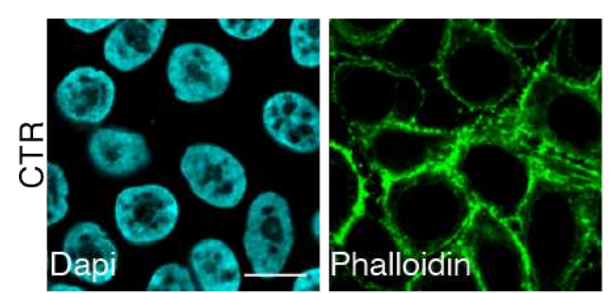

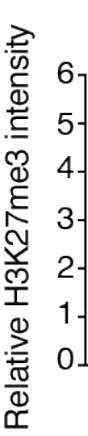

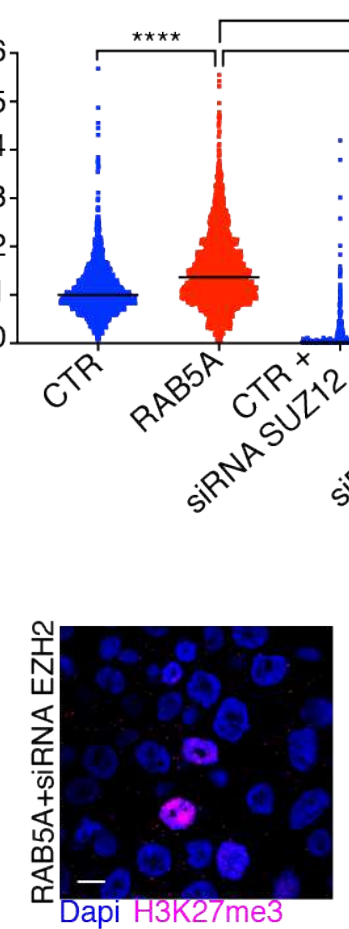

$\star \star \star \star$

$\pi * \star x$

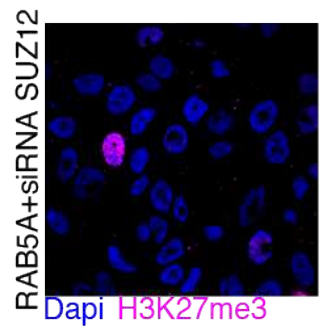

K
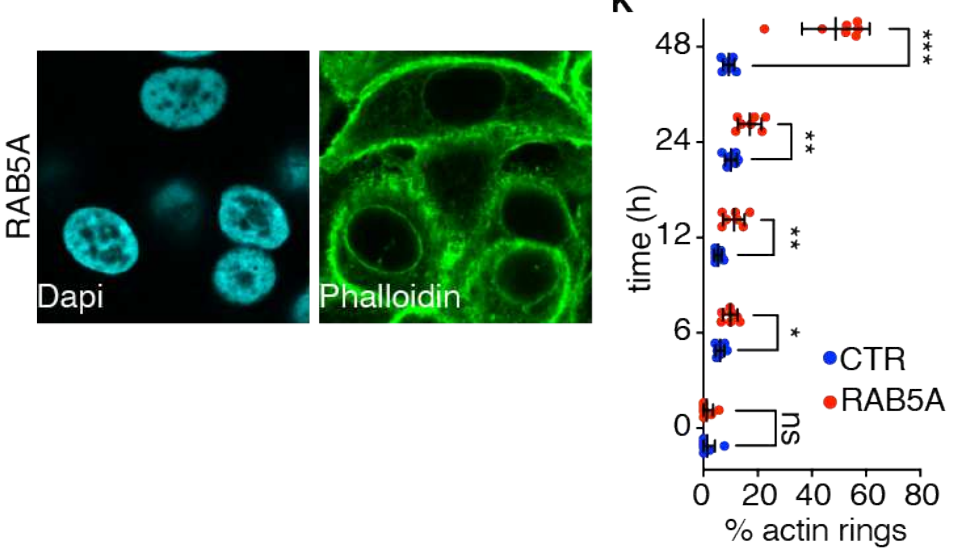
Figure 4. Tissue fluidification induces nuclear stiffness, heterochromatin reorganization, and actin remodeling

A. Left panel: representative map of the divergence of the velocity field in MCF10A RAB5A-expressing monolayer. The velocity field is obtained from PIV. Cold (warm) colors correspond to negative (positive) values of the divergence. Right panel: enlarged view of a small portion $(70 \times 70 \mu \mathrm{m})$ of the map shown on the left, centered on a selected nucleus (outlined in red). In this case, the velocity field (green arrows) points mainly towards the central cell. Locally, this corresponds to a negative value of the divergence and thus to a compressive deformation of the cell, which we systematically compare with the simultaneous nuclear deformation.

B-C. Root mean square value of the divergence of the velocity field obtained from PIV over the time window 4-20 $\mathrm{h}$ for MCF10A (B) and MCF10.DCIS.com (C) monolayers. Results are the mean \pm s.d.. [5 different FOVs (corresponding to roughly $1.2 \cdot 10^{4}$ cells)/ condition and 4 different FOVs (corresponding to roughly $8 \cdot 10^{3}$ cells)/ condition for MCF10A and MCF10.DCIS.com monolayers, respectively]. ${ }^{* * * *} \mathrm{p}<0.001, \mathrm{P}$ values, t-test (CTR versus RAB5A).

D-E. Nuclear strain rate $\dot{\gamma}_{n}$ as a function of the corresponding cell strain rate $\dot{\gamma}_{c}$ for MCF10A (D) and MCF10.DCIS.com (E) monolayers. $\dot{\gamma}_{n}$ is obtained from nuclear segmentation, while $\dot{\gamma}_{c}$ is estimated from the divergence of the velocity field measured with PIV. For clarity, data are grouped into evenly spaced bins along the horizontal axis. Symbols and error bars are the mean value and standard deviation of the $\dot{\gamma}_{n}-$ values of the data included in each bin, respectively. Straight lines are best fitting curves to the data with a linear model $\dot{\gamma}_{n}=s \dot{\gamma}_{c}$. Insets: relative nuclear stiffness, estimated as the inverse $1 / s$ of the slope obtained from the corresponding fitting model which is reported as mean \pm s.d. $(n=10$ randomly populated subsets of cells). ${ }^{* *} p<0.01,{ }^{* * *} p<0.0001, P$ values, T-test (CTR versus RAB5A).

F. Immunofluorescence images of doxycycline-treated control (CTR) and RAB5A-expressing (RAB5A) MCF10.DCIS.com monolayers stained with Dapi and anti-H3K27me3 antibody to detect nuclei and heterochromatin, respectively. Magnified images from the selected yellow boxes are shown. Scale bar 10 $\mu \mathrm{m}$.

G. Scatter plot of relative H3K27me3 intensity of control (CTR) and RAB5A-expressing (RAB5A) MCF10.DCIS.com monolayers silenced or not for EZH2 or SUZ12. Each dot represents a cell and the median is indicated. ( $n>1400$ cells/experimental condition for CTR and RAB5A, $n>400$ cells/experimental condition for siRNA-treated conditions). ${ }^{* * *} \mathrm{p}<0.0001, \mathrm{P}$ values, Kruskal-Wallis/Dunn's test.

H. Scatter plot of the ratio of H3K27me3 intensity at the nuclear central region over the nuclear periphery of control (CTR) and RAB5A-expressing (RAB5A) MCF10.DCIS.com. Each dot represents a cell and the mean \pm s.d. is indicated. ( $n>1000$ cells/experimental condition pooled from 3 independent experiments). ${ }^{* * * *} \mathrm{p}<0.0001, \mathrm{P}$ values, Mann-Whitney test.

I. Immunofluorescence images of control empty vector (CTR) and RAB5A-expressing (RAB5A) MCF10.DCIS.com monolayers silenced for EZH2 or SUZ12 and stained with DAPI and anti-H3K27me3 antibody to detect nuclei and heterochromatin, respectively. Scale bar $10 \mu \mathrm{m}$.

J. Immunofluorescence images of control (CTR) and RAB5A-expressing (RAB5A) MCF10.DCIS.com monolayers stimulated with doxycycline to induce RAB5A expression, fixed at various time points and stained with phalloidin to detect F-actin. Representative images of duplicate experiments are a magnification of $48 \mathrm{~h}$ time points to show actin ring structures. Scale bar $20 \mu \mathrm{m}$.

K. Scatter plot of the percentage of actin ring structures/FOV at the indicated time points. Data are expressed as the mean \pm s.d. $\left(n=2\right.$ independent experiments). ns $>0.999,{ }^{*} p<0.05,{ }^{* *} p<0.01,{ }^{* * *} p<$ $0.001, \mathrm{P}$ values, each-pair Mann Whitney test (CTR versus RAB5A). 
A
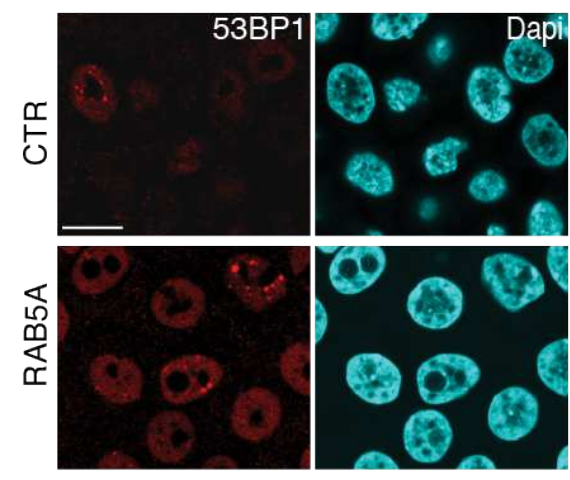

B

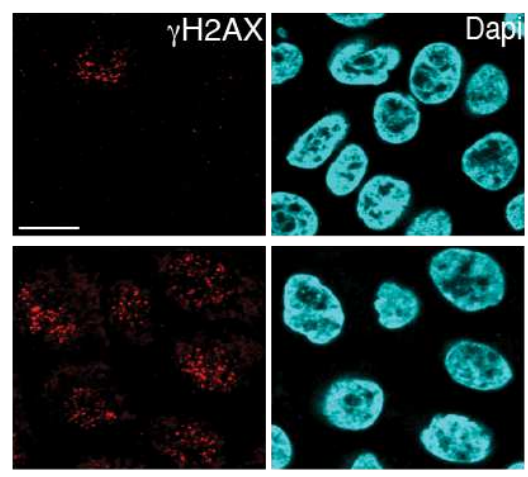

C

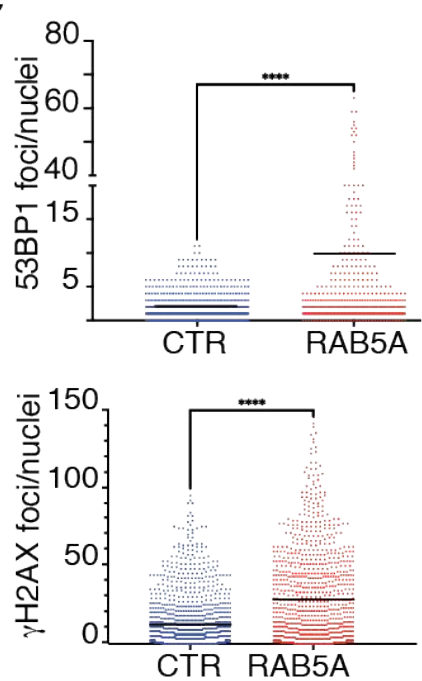

E
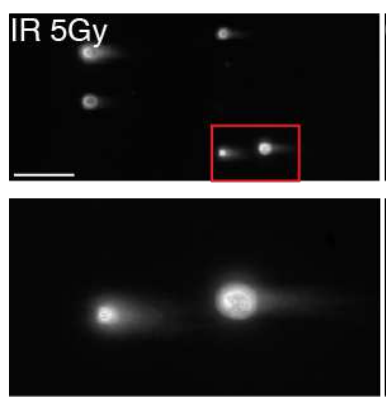
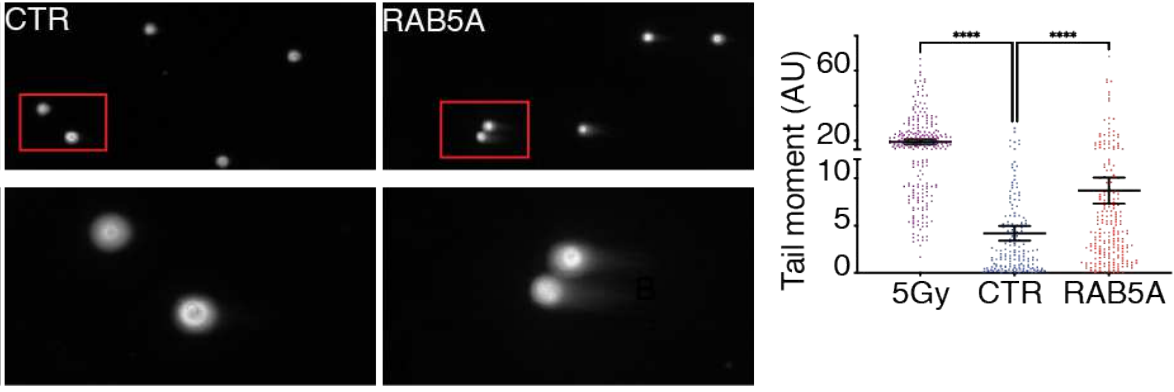

\section{Figure 5. Endocytic-dependent tissue fluidification results in DNA damage}

A-B. Images of control and RAB5A-MCF10.DCIS.com monolayers stained with the indicated antibodies. Scale bar, $20 \mu \mathrm{m}$.

C. The scatter plot shows the mean of 53BP1 foci/nuclei or $\gamma \mathrm{H} 2 \mathrm{AX}$ foci/nuclei ( $n>150$ out of three independent experiments). ${ }^{* * * *} p<0.001, P$ values, unpaired Mann Whitney $t$-test (CTR versus RAB5A).

D. Representative images of neutral comet assay carried out in MCF10.DCIS.com monolayer cells expressing RAB5A. Control cells irradiated (IR 5Gy) or not (CTR) are also shown. Scale bar, $100 \mu \mathrm{m}$.

E. The scatter plot shows the quantification of DNA damage by tail moment analysis. Horizontal bars indicate the means and s.d. of at least 2 independent experiments; more than 100 cells per sample were scored. ${ }^{* * *} p<0.001, P$ values, one-way ANOVA. 
A

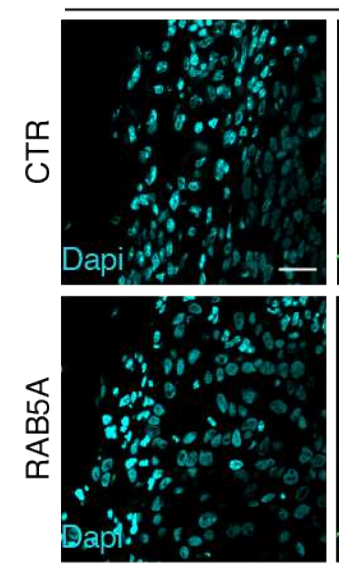

C

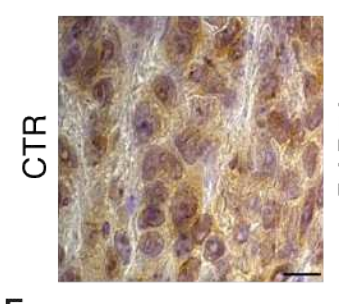

E
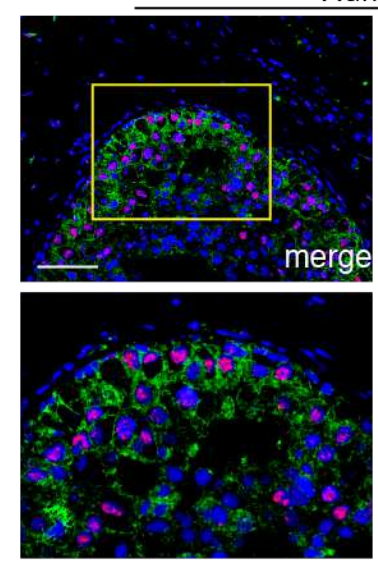

G
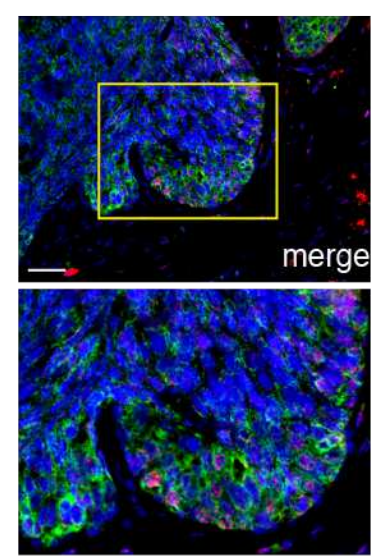

Human early breast cancer lesion
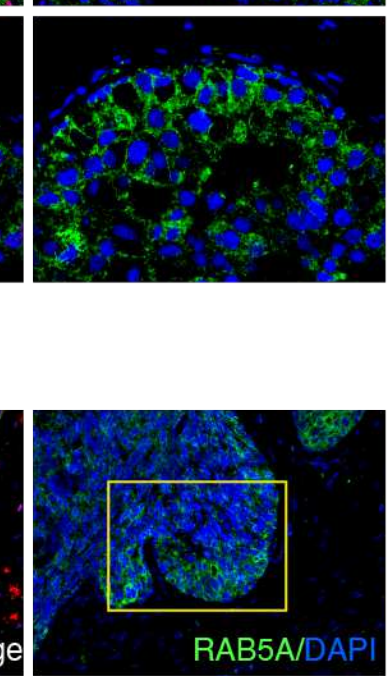

murine xenograft
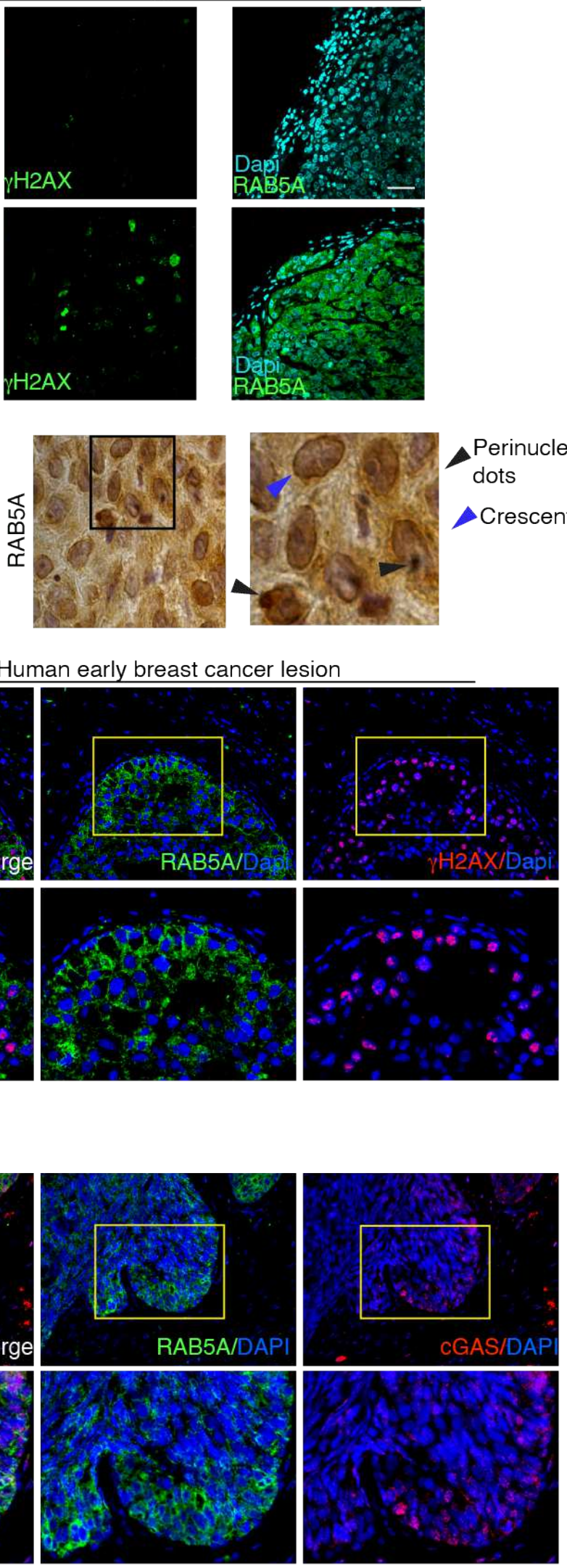

B

Figure 6

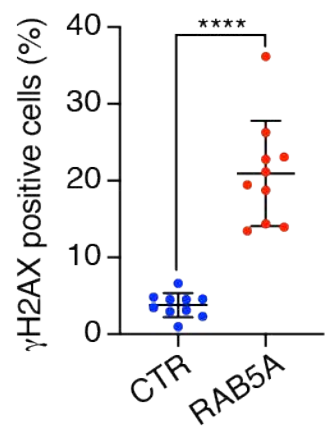

D

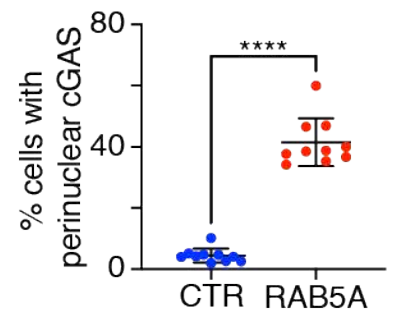

F

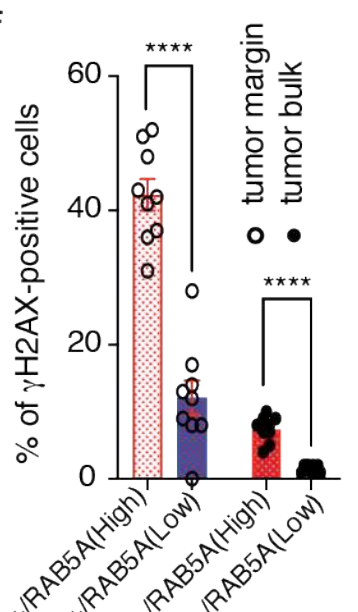

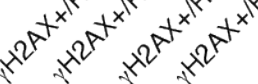

$\mathrm{H}$

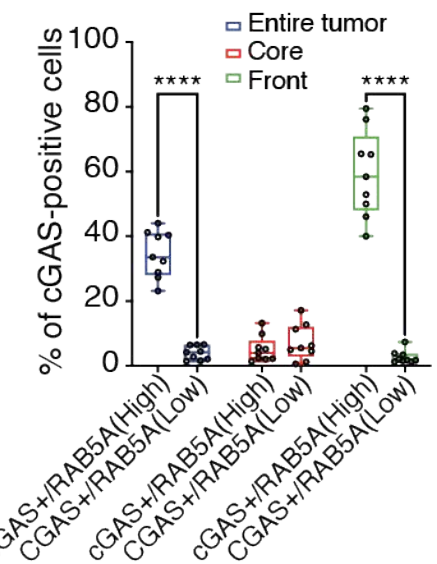


Figure 6. Increased RAB5A-expression, cGAS perinuclear accumulation, and DNA damage in human invasive ductal carcinoma

A. Immunofluorescence images of control (CTR) and RAB5A-expressing (RAB5A) MCF10.DCIS.com injected into mammary fat pads of immunocompromised mice. After one week, mice were fed with doxycycline to induce RAB5A expression. Four weeks after doxycycline treatment, primary tumors were isolated, fixed and stained with Dapi and $\gamma \mathrm{H} 2 \mathrm{AX}$ to detect nuclei and DNA damage foci, respectively. The samples were also stained with Dapi and RAB5A. Scale bar $100 \mu \mathrm{m}$.

B. Scatter plot of the percentage of $\gamma \mathrm{H} 2 \mathrm{AX}$ positive cells/FOV. Data are mean $\pm \mathrm{s}$.d. $(n=2$ independent experiments, with 5 mice/experiment). ${ }^{* * *} p<0.0001, P$ values, each-pair Welch's test (CTR versus RAB5A).

C. Immunohistochemical analysis of cGAS expression and localization in control (CTR) and RAB5Aexpressing (RAB5A) MCF10.DCIS.com injected into mammary fat pads of immunocompromised mice. After one week, mice were fed with doxycycline to induce RAB5A expression. Four weeks after doxycycline treatment, primary tumors were isolated, fixed and stained with a specific cGAS antibody. The right panel shows a higher magnification of the boxed region to display enhanced levels of cGAS with perinuclear dotted (black harrow-heads) or crescent-like distribution (blue harrow-head). Scale bar $80 \mu \mathrm{m}$.

D. Scatter plot of the percentage of cells with perinuclear cGAS enrichment is expressed as the mean \pm s.d. (at least $n=5 \mathrm{FOV} /$ experimental conditions in two independent experiments). ${ }^{* * *} p<0.0001$, P values, each-pair Mann Whitney test (CTR versus RAB5A).

E. Representative multiplex immunohistochemistry/Immunofluorescence (mIHC/IF) of RAB5A, $\gamma \mathrm{H} 2 \mathrm{AX}$ and Dapi in human Ductal breast Carcinoma in Situ (DCIS). Magnified images from the selected yellow boxes are shown. Scale bar $150 \mu \mathrm{m}$.

F. Box and scatter plots showing the percentage of $\gamma \mathrm{H} 2 \mathrm{AX}$-positive nuclei in cells that express high ( $>2$ on a scale from $0.1,2,3)$ or low I $(<2$ on a scale from $0.1,2,3)$ levels of RAB5A in the tumor bulk or margin (defined by an automated, operator-independent analysis of all cell nuclei at a set distance from the tumor edge versus those nuclei present in the tumor bulk-see methods for details and Movie S9). The areas of 9 independent DCIS were analyzed with more than 200 nuclei per area. ${ }^{* * *} P<0.0001$, Nested 1 way ANOVA test.

G. Representative multiplex immunohistochemistry/immunofluorescence (mIHC/IF) of RAB5A, cGAS , and Dapi in human Ductal breast Carcinoma in Situ (DCIS). Magnified images from the selected yellow boxes are shown. Scale bar $300 \mu \mathrm{m}$.

H. Box and scatter plots showing the percentage of cGAS-positive cells that express high ( $>2$ on a scale from $0.1,2,3)$ or low $(<2$ on a scale from $0.1,2,3)$ levels of RAB5A in the tumor bulk, or the margin or the whole tumor (the margin and tumor bulk were defined by an automated, operator-independent analysis of all cell nuclei at a set distance from the tumor edge versus those nuclei present in the tumor bulk-see methods for details). The areas of 9 independent DCIS were analyzed with more than 200 nuclei per area. ${ }^{* * *} \mathrm{P}<0.0001$, Mann-Whitney two-tailed t-test. 


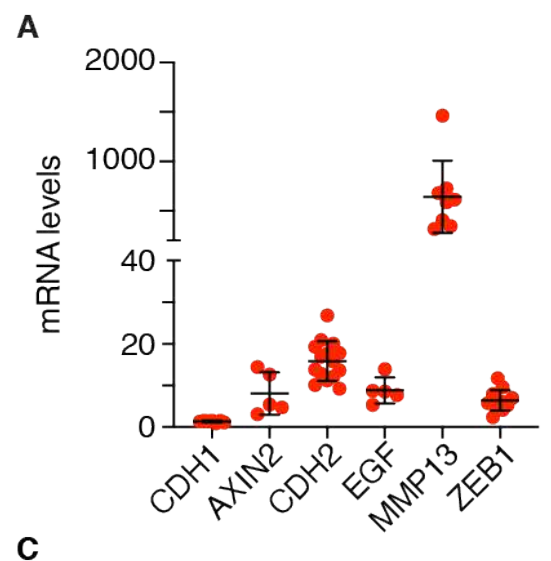

B

Figure 7
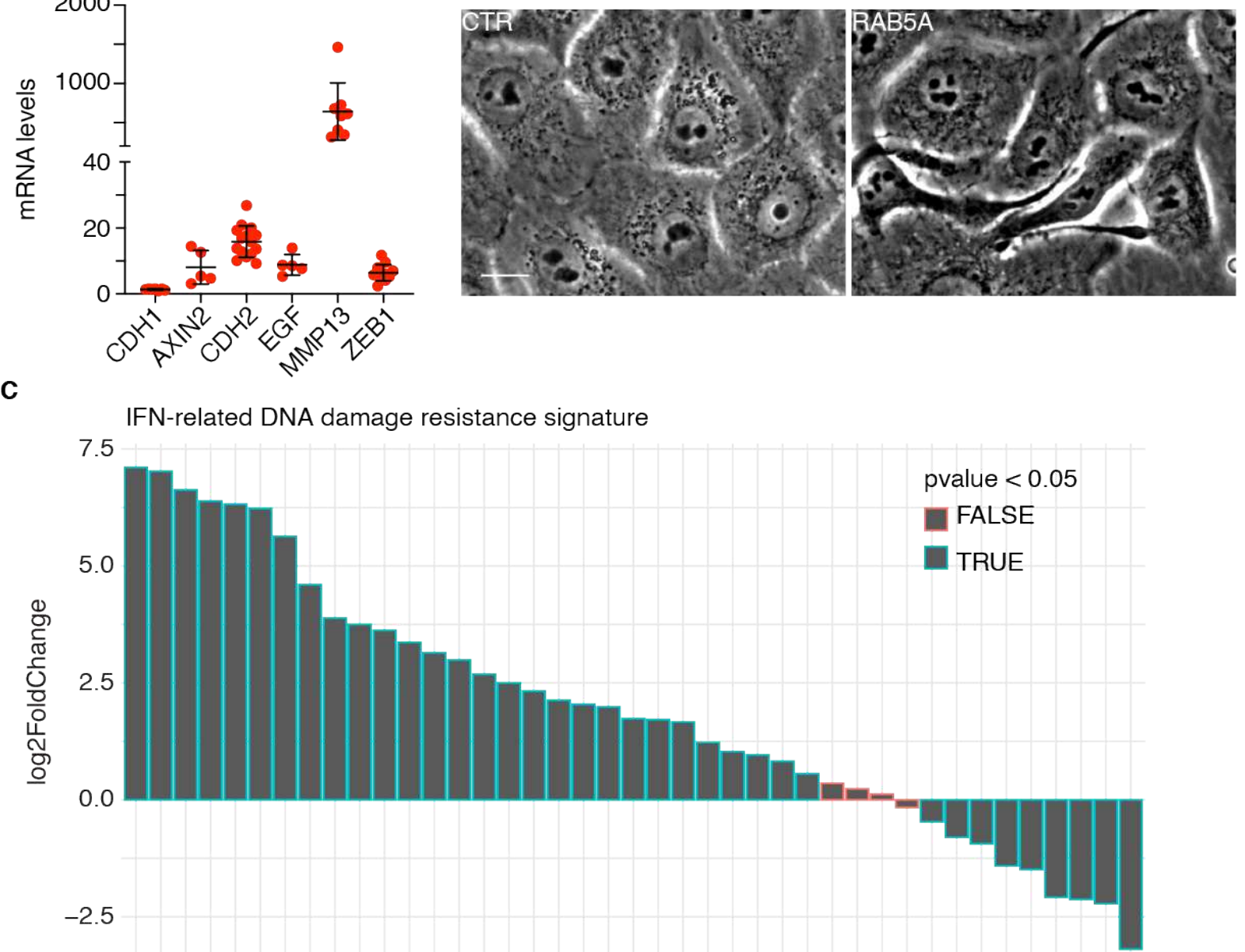

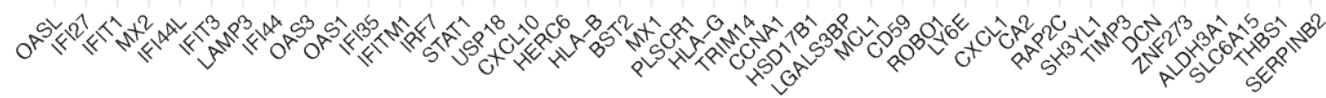

D
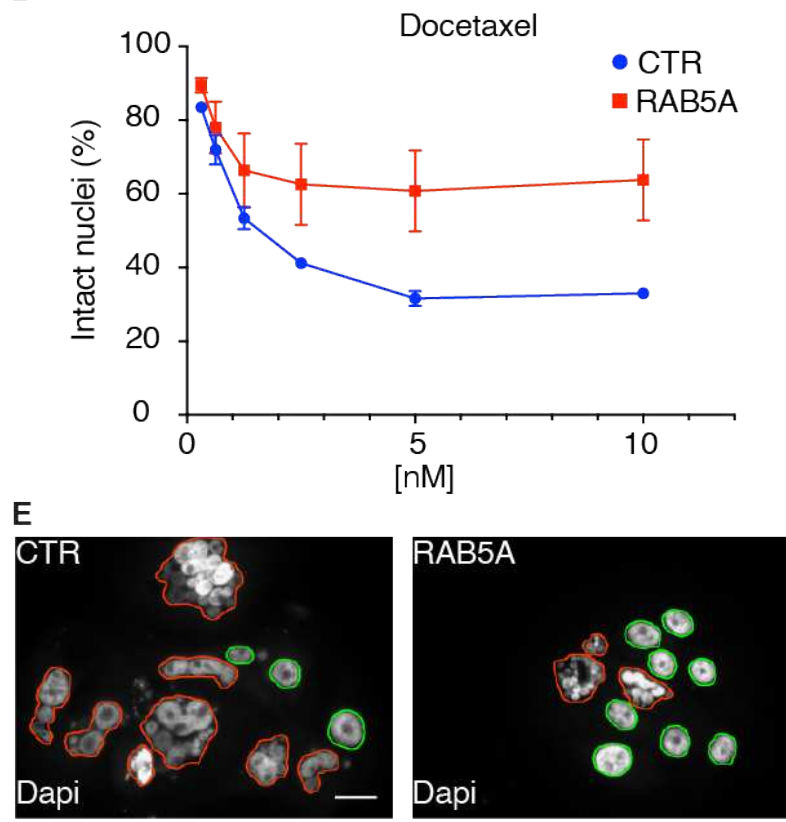

$\mathbf{F}$

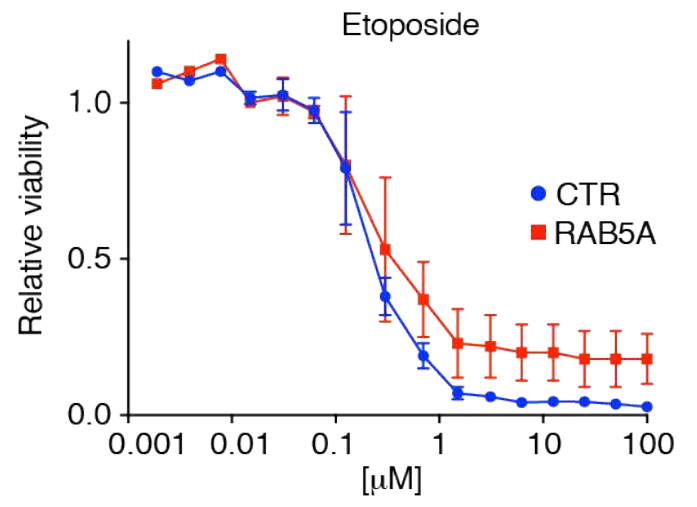


Figure 7. Tissue fluidification enhances plastic EMT traits and chemoresistance A. Scatter pots of the mRNA expression levels of CDH1, AXIN2, CDH2, EGF, MMP13, and ZEB1 determined by qRT-PCR in RAB5A-expressing MCF10.DCIS.com monolayers over control cells. Data are the mean \pm s.d. (at least $n=5$ independent experiments). Values were normalized to the controls of each experiment.

B. Phase-contrast images of control empty vector (CTR) and RAB5A-expressing (RAB5A) MCF10.DCIS.com monolayer. Scale bar, $20 \mu \mathrm{m}$.

C. Quantitative changes in the expression of the INF-related DNA damage resistance signature genes in RAB5A-expressing MCF10.DCIS monolayers with respect to their respective controls. The log2Fold Change is plotted on the $x$-axis and the significance (Wald test $p$-value) is defined by the color code of the outline.

D. Percentage of intact nuclei after treatment with the indicated doses of docetaxel in control (CTR) and RAB5A-expressing (RAB5A) MCF10.DCIS.com cells. Data are the mean \pm s.d. $(n=3$ independent experiments).

E. Representative images of control empty vector (CTR) and RAB5A-expressing (RAB5A) MCF10.DCIS.com nuclei stained with Dapi in the presence of $10 \mathrm{nM}$ Docetaxel. Intact and aberrant polynucleated cell nuclei are indicated by green and red contours. Scale bar $10 \mu \mathrm{m}$.

F. Dose-dependent analysis of cell proliferation of control (CTR) and RAB5A-expressing (RAB5A) MCF10.DCIS.com treated with the indicated concentrations of Etoposide. Data are the mean \pm s.d. $(n=3$ independent experiments). 


\section{Supplementary Files}

This is a list of supplementary files associated with this preprint. Click to download.

- MovieS5.mp4

- Movies8.mp4

- MovieS4.mp4

- MovieS7.mp4

- MovieS6.mp4

- MovieS1.mp4

- Movies3.mp4

- MovieS9.mp4

- Movies2.mp4

- supplementaryinformation.pdf 